\title{
Predicción del cheapest to deliver en los contratos de futuros sobre bono nocional de corto, mediano y largo plazo*
}

\section{Prediction of the cheapest to deliver in futures contracts on short, medium and long term notional bonds}

Kimberly Rojas-Silva*

* $\quad$ Este documento fue presentado como requisito para obtener el título de magíster en Finanzas en la Universidad Externado de Colombia. Se agradece especialmente la asesoría de Javier Sandoval en la estructuración y el desarrollo de esta investigación. De igual manera, se agradecen los valiosos comentarios y observaciones de Juan Sebastián Lemus.

Las opiniones expresadas en este documento son responsabilidad del autor y no comprometen al Banco de la República ni a su Junta Directiva. Cualquier error u omisión en el contenido de este texto es responsabilidad exclusiva del autor.

** Magíster en Finanzas, profesional especializado. Mesa de Dinero, Banco de la República, Bogotá (Colombia). [krojassi@banrep.gov.co].

Artículo recibido el 10 de noviembre de 2018.

Aceptado el 01 de diciembre de 2018.

Para citar este artículo:

Rojas-Silva, K. (2018). Predicción del cheapest to deliver en los contratos de futuros sobre bono nocional de corto, mediano y largo plazo. ODEON, 15, 73-137.

DOI: https://doi.org/10.18601/17941113.n15.04 


\section{Introducción}

Este estudio presenta los resultados de la implementación del modelo de Ho-Lee para la valoración de bonos, y el cálculo del cheapest to deliver (CTD) en los contratos de futuro sobre bono nocional del mercado de deuda pública colombiano.

En septiembre de 2008, la Bolsa de Valores de Colombia (BVC) lanzó los contratos de futuros sobre títulos de deuda pública, con el fin de profundizar y complementar el mercado de contado de deuda pública, y permite realizar operaciones de cobertura de tasa de interés, así como estrategias de especulación en los movimientos de tasa de interés. Este producto fue el primer derivado estandarizado que se negoció en el mercado de capitales colombiano y buscaba, además, aumentar la eficiencia de la administración de portafolios, y posibilitar el arbitraje y la formación de precios justos.

En comparación con países desarrollados, el mercado de derivados estandarizados en Colombia aún es pequeño e incipiente. El estudio de estos productos ayuda a comprender mejor las dinámicas del mercado local y contribuye a su profundización.

\section{Mercado de futuros}

En Colombia se negocian varios tipos de futuros de tasas de interés, entre ellos futuros sobre índices como la inflación (índice de precios al consumidor - IPC) y el Indicador Bancario de Referencia (IBR), y otros cuyos subyacentes corresponden a bonos de deuda pública. En esta última categoría se encuentran los futuros de Títulos de Deuda Pública (TES) de referencias específicas, y los futuros de TES nocionales. Por una parte, los futuros de TES de referencias específicas tienen como activo subyacente a un título "específico" de renta fija en pesos emitido por el Gobierno.

Por otra parte, los futuros de TES nocionales corresponden a aquellos que se negocian sobre un bono teórico, que para efectos de la negociación sustituye al conjunto de bonos de una canasta de entregables. Los bonos que constituyen la canasta de entregables son activos similares y se relacionan con el nocional a través de un factor de conversión. Dependiendo del plazo del bono teórico, los futuros de TES se clasifican en futuros de corto ( 2 años), mediano (5 años) y largo plazo (10 años), y para todos el subyacente es un título TES clase B tasa fija en pesos con pago de cupón anual.

La presente investigación analiza el comportamiento de los futuros de TES nocionales y el CTD, que corresponde al bono que el vendedor entregaría a su 
contraparte el día de vencimiento del contrato. No obstante, durante la vida del contrato de futuro el CTD puede cambiar cuando existen cambios relativos en los niveles de tasa de los bonos entregables, en la forma de curva de rendimientos o en las tasas repos para varios de estos bonos, y el CTD sería aquel que presente:

- La mayor duración si la tasa de interés de mercado es mayor que el cupón del nocional.

- La menor duración si la tasa de interés de mercado es menor que el cupón del nocional.

Dados los cambios de duración del entregable, el comprador se vería expuesto a cambios en el perfil de riesgo de su portafolio, que podrían ir en detrimento de su rendimiento y las políticas fijadas por su entidad. Por tanto, es conveniente para los inversionistas contar con una herramienta que permita conocer cuál de los entregables corresponderá al CTD o, al menos, indicar cuál de los entregables tiene la mayor probabilidad de serlo (Choudhry, 2010).

Henrard (2006) desarrolla una metodología de valoración de futuros basada en opciones, en la que se prueban con casos prácticos las condiciones bajo las cuales cambia el CTD y el impacto de dicho cambio en el perfil de riesgo del tenedor.

Los contratos que trata el presente estudio corresponden a los futuros sobre bonos nocionales que se negocian en la BVC. En estos contratos, el vendedor (parte corta) se compromete entregar al comprador (parte larga) un bono nocional en una fecha futura predefinida (fecha de vencimiento del futuro o de delivery).

Durante la negociación se transa el bono nocional que es un bono teórico emitido a la par ${ }^{1}$. Para efectos del cumplimiento, el contrato de futuro tiene como subyacente una canasta de bonos entregables con características similares, y son títulos TES clase B tasa fija en pesos, que se relacionan con el bono teórico a través de un factor de conversión (Bolsa de Valores de Colombia, 2014).

A pesar de que la parte corta puede elegir cualquier bono dentro de una canasta de entregables en la fecha de delivery, este preferirá entregar el CTD que es aquel cuyo costo de entrega sería el menor al calcular la siguiente expresión:

Precio limpio del Bono $_{i}-\left(\right.$ Precio del Futuro $*$ Factor de Conversión $\left._{i}\right)$

1 El día de vencimiento de cada contrato. 
La BVC tiene un ciclo de vencimientos trimestrales en los meses de marzo, junio, septiembre y diciembre. Además de vencimientos trimestrales, la BVC también cuenta con vencimientos mensuales. En todo momento hay disponibles para la negociación cuatro contratos: tres con vencimientos mensuales más cercanos y uno trimestral dentro del ciclo hasta seis meses. Cuando un contrato llega al vencimiento, se genera uno nuevo para mantener la estructura de vencimientos (Bolsa de Valores de Colombia, 2014).

\subsection{Características de los contratos de futuros sobre bono nocional}

El mnemotécnico de los contratos de futuros es el siguiente (tabla 1):

Tabla 1: Descripción del mnemotécnico de los contratos de futuros de TES

\begin{tabular}{|c|l|l|l|l|}
\hline \multicolumn{5}{|c|}{ TEMZ08F } \\
\hline \multicolumn{1}{|c|}{ TE } & \multicolumn{1}{|c|}{ M } & \multicolumn{1}{|c|}{$\mathbf{Z}$} & \multicolumn{1}{c|}{$\mathbf{0 8}$} \\
\hline $\begin{array}{l}\text { Contrato sobre un } \\
\text { título de deuda pú- } \\
\text { blica colombiana }\end{array}$ & $\begin{array}{l}\text { Plazo del contrato. S: } \\
\text { corto plazo, M: me- } \\
\text { diano plazo y L: largo } \\
\text { plazo }\end{array}$ & $\begin{array}{l}\text { Mes en que vence } \\
\text { el contrato. En este } \\
\text { ejemplo corresponde } \\
\text { al mes de diciembre }\end{array}$ & $\begin{array}{l}\text { Año de vencimiento } \\
\text { del contrato. Para es- } \\
\text { te ejemplo es el 2008 }\end{array}$ & $\begin{array}{l}\text { Indica que es } \\
\text { contrato de } \\
\text { futuro. }\end{array}$ \\
\hline
\end{tabular}

Fuente: elaboración propia.

Estos contratos se negocian por precio y tienen un valor nominal de $\$ 250.000 .000$, con un tick de precio de 0,005 unidades que es equivalente a $\$ 12.500$. La convención establece que el día de vencimiento de un contrato es el primer viernes del mes, pero el último día de negociación es el miércoles de esa semana (Bolsa de Valores de Colombia, 2014).

La liquidación de las operaciones se realiza por entrega física del activo subyacente en la fecha de delivery, y está sujeta al procedimiento establecido por la Cámara de Riesgo Central de Contraparte de Colombia (CRCC). Además, estos contratos son marked to market, es decir que las posiciones abiertas al final del día son valoradas al precio de liquidación por la Cámara de Riego Central de Contraparte de Colombia (CRCC) (2014).

\subsection{Evolución del mercado colombiano de futuros sobre bono nocional}

Las operaciones del mercado de futuros de TES han presentado un comportamiento mixto, caracterizado por periodos de bajo dinamismo, seguidos por intervalos 
con más contratos negociados. El periodo comprendido entre septiembre de 2008 y diciembre 2014 se puede dividir en tres subperiodos: i) de septiembre-2008 a diciembre-2010: en este periodo se lanza el producto y corresponde al de menor número de contratos pactados, los cuales oscilan entre 70 y 8726 mensuales; ii) de enero-2011 a diciembre-2012: el mercado se consolida y aumentan las transacciones, los contratos pactados oscilan entre 1428 y 29.584 mensuales; y iii) de enero-2013 a diciembre-2014: se presenta el máximo volumen mensual transado y se reduce significativamente su dinamismo desde mayo-2013 hasta el final del periodo (máx.: 42.263 y mín.:1769). Cabe resaltar que no hay contratos pactados desde diciembre del 2014; según algunos agentes consultados, este producto tuvo poca acogida por la mayor complejidad que implica el manejo de una canasta de entregables, y por los cambios que tiene el CTD durante la vigencia del contrato; además, luego de la introducción de contratos sobre referencias específicas $^{2}$, el interés sobre los nocionales fue aún menor. Durante el periodo septiembre-2008 a diciembre-2014, los agentes prefirieron los futuros de corto y mediano plazo, con una participación de 44,66 y 38,92\%, respectivamente, sobre el total pactado (figura 1).

Figura 1: Evolución del mercado de futuros de TES (periodo 2008-2013)

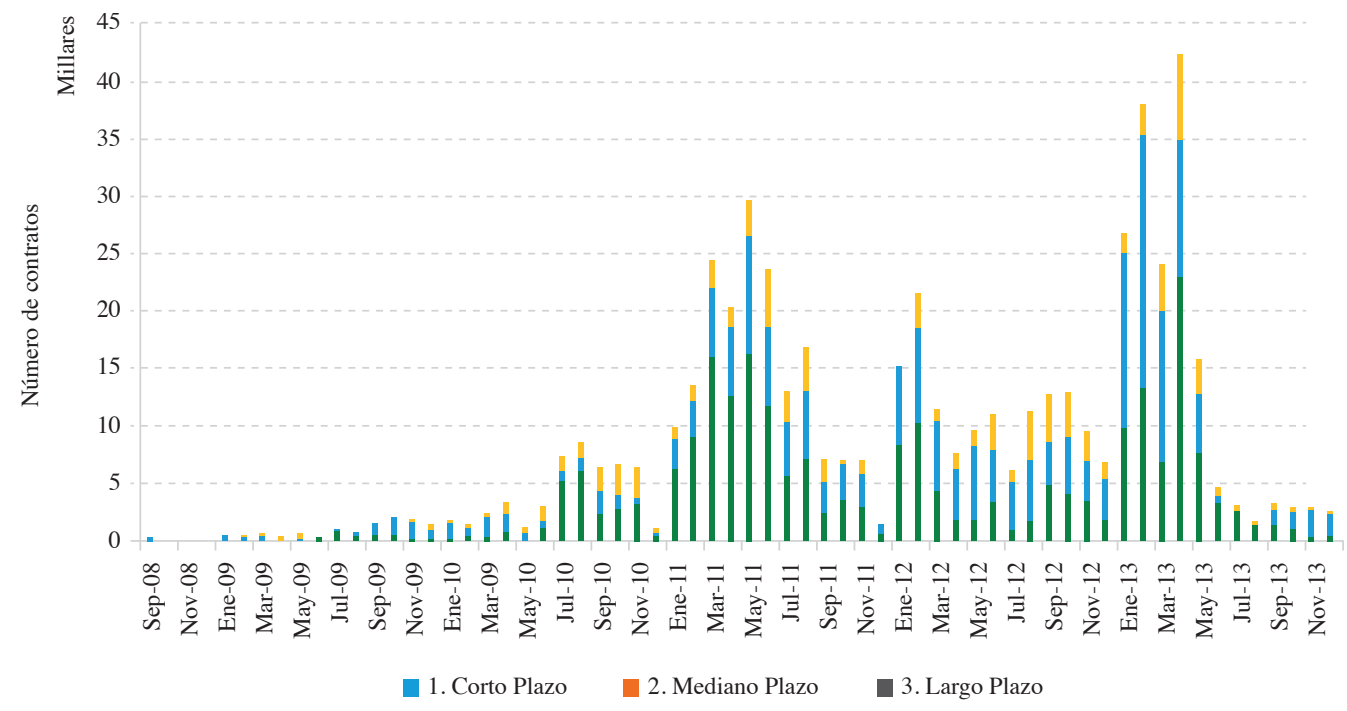

2 Se empezaron a negociar desde abril de 2013. 
Con base en los precios de cierre de las operaciones de compra y venta de TES en pesos, se aplicó el modelo discreto de Ho-Lee para hallar el precio de los bonos y el de los contratos de futuro en la fecha de delivery. Con esta información fue posible identificar el CTD. Finalmente, un análisis de sensibilidad determinó cómo se ve afectado el CTD ante cambios en la curva cero cupón (CCC) y en la volatilidad.

\section{Estado del arte}

La presente investigación siguió la metodología de van Straaten (2009), con el objeto de identificar qué bono presentaba la mayor probabilidad de convertirse en el CTD en la fecha de delivery del contrato de futuro.

van Straaten (2009) utilizó el modelo de Ho-Lee para hallar el precio de los bonos entregables y del contrato de futuro desde la fecha de interés hasta la fecha de delivery. El modelo utilizado permitió construir un árbol binomial que replica el comportamiento de la CCC durante el periodo de análisis. Con base en las tasas del árbol Ho-Lee se calculó el precio de los bonos entregables y del futuro en la fecha de delivery, y con esta información fue posible determinar el bono CTD. El autor concluyó que el modelo es satisfactorio, con una diferencia máxima entre el precio teórico y el de mercado de 0,6\%. Adicionalmente, obtuvo la probabilidad de que cada entregable se convirtiera en el CTD a lo largo del árbol binomial (van Straaten, 2009).

Dado que el CTD puede cambiar de acuerdo con los movimientos de las tasas de interés durante la vigencia del contrato, se dificulta la aplicación de estrategias de cobertura. Al respecto Grieves et al. (2010) estudiaron el problema de cobertura de tasas de interés utilizando notas y bonos del Tesoro estadounidense. Con base en la premisa de que solo dos de los entregables son relevantes en el problema de cobertura ${ }^{3}$ (el de mayor y menor duración), los autores evaluaron la eficacia empírica de modelos con dos bonos relevantes. En esta investigación se encontró que el modelo se desempeña muy bien para el contrato sobre la nota mientras que presenta un desempeño muy pobre para el contrato sobre el bono, y concluyeron que las diferencias en el desempeño están relacionadas con la forma de la curva de rendimientos (Grieves et al., 2010).

3 Esta afirmación la extrajeron los autores Grieves y Marcus (2005, pp. 70-76). 
Ho y Lee (1986) desarrollaron un modelo para pronosticar los posibles movimientos de la CCC, motivados por el incremento en el uso de derivados de tasas de interés y bajo la premisa de que estos productos dependen del comportamiento de la CCC. El interés por calcular primero esta curva radica en que a partir de ella es posible hallar el precio de cualquier derivado de tasa de interés y, por tanto, la metodología no estaría supeditada a una sola clase de activo contingente (Ho y Lee, 1986).

Dentro de los trabajos que han aplicado el modelo de Ho-Lee se puede citar el de Zazaravaka (2010), en el que se resaltó que el modelo depende exclusivamente de la volatilidad y de la probabilidad neutral al riesgo. Destacó además que permite tener en cuenta los posibles riesgos de tasas de interés debido a perturbaciones económicas, a diferencia de otros modelos de tasa de interés constante como el de Black-Scholes o el de Cox-Ross-Rubinstein. En este documento, el autor probó que en el modelo de Ho-Lee se excluyen todas las posibilidades de arbitraje, y que las características de las probabilidades neutrales al riesgo aseguran que el mercado sea completo. No obstante, se mencionaron desventajas tales como la posibilidad de evidenciar tasas negativas y una menor flexibilidad al considerar la volatilidad de las tasas de interés como constante

El algoritmo de Leippold y Wiener (1992) buscó replicar el comportamiento de cualquier activo de tasa de interés. En su desarrollo los autores enfatizaron que una de las mayores ventajas de la metodología es que, en la fecha inicial del árbol de tasas de interés, la CCC coincide con la curva del mercado, por lo que el reto corresponde a la modelación de la incertidumbre de la CCC durante el periodo de interés.

De otra parte, Grant y Vora (2001) resaltaron la complejidad de la implementación discreta del modelo Ho-Lee, que por lo general requiere de métodos numéricos e inducción. En su trabajo, los autores identificaron expresiones recursivas en cada nodo, utilizando modelos binomiales y simulación de Monte Carlo para hallar las tasas de interés de corto plazo.

Flesaker (1993) realizó pruebas empíricas sobre el modelo de Heath, Jarrow y Morton, utilizando una volatilidad constante 4 y el método de momentos generalizado con datos diarios de los futuros y opciones sobre futuros de Eurodólar. Los resultados muestran que el modelo no se ajusta a las dinámicas de los

4 Este modelo corresponde al límite en tiempo continuo del modelo originalmente desarrollado por Ho y Lee. 
contratos de opciones sobre futuros, sobrevalorando las opciones de corto plazo y subvalorando las de largo plazo; al parecer, tales hallazgos obedecen a que se omitieron consistentemente el riesgo de crédito y la prima por riesgo de crédito 5 .

Dada la importancia de capturar el comportamiento de las tasas de interés al valorar activos contingentes y aplicar estrategias de cobertura, Chan, Karolyi, Longstaff, Sanders y Longstaff (2014) realizaron la estimación y comparación de ocho modelos en tiempo continuo de tasas de interés de corto plazo. En este documento se encontró que los modelos más exitosos en el ajuste, utilizando datos del rendimiento de los Treasury Bills, fueron aquellos que permiten cambios en la volatilidad de la tasa de interés. Particularmente, se encontró una alta dependencia entre la volatilidad y el nivel de la tasa de interés, razón por la cual modelos comúnmente usados como el de Vasicek (1977) y Cox, Ingersoll y Ross (1985) presentaron un peor desempeño al compararse con modelos menos conocidos como el de Dothan (1978) y Cox, Ingersoll y Ross (1980).

\section{Metodología}

En la presente sección se describen las generalidades de las técnicas aplicadas a lo largo del cálculo del CTD. En el desarrollo del estudio se siguió un proceso similar al aplicado por van Straaten (2009), en el que se requiere primero calcular una CCC, con base en la que se construye el árbol de tasas de interés de corto plazo. Para calcular la CCC se utilizó el bootstrapping y el modelo de Ho-Lee (1986) para construir el árbol binomial discreto de tasas de interés. Posteriormente, se calcularon los factores de descuento usando la información de CCC y el árbol de tasas Ho-Lee, con ellos fue posible calcular el precio limpio de los bonos y del contrato de futuro en la fecha de delivery. Finalmente, se halló el CTD utilizando los datos ya mencionados.

\subsection{Bootstrapping e interpolación}

El bootstrapping consiste en hallar de forma sistemática y recursiva las tasas cero cupón a partir del precio de los bonos de deuda pública disponibles en el mercado. Dado que un bono con cupones puede descomponerse en un conjunto

5 El índice subyacente de los contratos Eurodólar es la Libor a tres meses, que involucran la prima de riesgo por encima de las tasas de los Treasury Bills. 
de bonos a descuento que corresponden tanto a los intereses como al principal, el precio del bono debe ser la suma de los precios de bonos a descuento. Así, si existen bonos para todos los plazos de la curva de rendimientos, la CCC puede hallarse utilizando el siguiente procedimiento (Julio, Mera y Revéiz, 2002):

- La tasa spot a un periodo es igual al yield de un bono a un periodo.

- Se descuenta el primer cupón del bono con vencimiento a dos periodos y se sustrae del precio del bono a dos periodos, este valor corresponde al valor de mercado del principal y el cupón que se pagan en el segundo periodo. Una vez calculado este valor, es posible calcular la tasa de descuento que iguala el valor presente al del cupón y del principal que se pagan en el segundo periodo. Esta tasa corresponde a la tasa spot a dos años.

- El proceso se repite de la misma forma para hallar secuencialmente las tasas spot para todos los plazos.

La CCC que se obtiene es libre de arbitraje para el conjunto de bonos usados, por esto es preciso utilizar los títulos de la canasta de entregables dentro del cálculo de la misma. Se escogió esta técnica debido a que garantiza que el precio teórico de los bonos será igual al observado, en el punto inicial del árbol (ecuación 2, método Bootstrapping).

$$
\begin{gathered}
J\left(\vec{c}, \overrightarrow{t_{C}}, t\right)=\sum_{i=1}^{N} c_{t i} e^{-\left(t_{i}-t\right) z\left(t, t_{i}\right)}+100 * e^{-\left(t_{N}-t\right) z\left(t, t_{N}\right)} \\
Z\left(t, t_{N}\right)=\frac{-1}{t_{N}-t} \operatorname{In}\left(\frac{J\left(\vec{c}, \overrightarrow{t_{o}} t\right)-\sum_{i=1}^{N-1} c_{t i} e^{-\left(t_{i}-t\right) z\left(t, t_{i}\right)}}{100+c_{t_{N}}}\right)
\end{gathered}
$$

Se empleó el método de interpolación linear on the log of discount factors para completar la información de tasas que no era posible conocer con los bonos disponibles en el mercado (Hagan y West, 2008). Se escogió este método debido a que la tasa forward de la curva es positiva y constante, es muy estable, fácil de implementar y comúnmente usada en la construcción de curvas de tasas de interés (ecuación 3, método de interpolación linear on the log of discount factors).

$$
Z(t)=\frac{t-t_{i}}{t_{i+1}-t_{i}} \frac{t_{i+1}}{t} Z\left(t_{i+1}\right)+\frac{t_{i+1}-t}{t_{i+1}-t_{i}} \frac{t_{i}}{t} Z\left(t_{i}\right)
$$




$$
f(t)=\frac{Z\left(t_{i+1}\right) t_{i+1}-Z\left(t_{i}\right) t_{i}}{t_{i+1}-t_{i}}
$$

La ecuación 4 corresponde a la tasa forward de la curva hallada con el método de interpolación linear on the log of discount factors.

\subsection{Modelo Ho-Lee para la valoración de productos de renta fija}

El modelo Ho-Lee se aplica para calcular el CTD en la fecha de delivery. Este toma la estructura temporal de tasas de interés (ETTI) como un dato de entrada en la fecha inicial $(t=0)$ y deriva los movimientos subsecuentes de la misma hasta el vencimiento del activo. Los movimientos de la curva no deben permitir la existencia de oportunidades de arbitraje, de esta forma el modelo es consistente con uno de equilibrio general. El modelo valora los activos contingentes por medio de los cambios observados en la ETTI, lo que permite aplicarlo a varias clases de derivados de tasas de interés (Ho y Lee, 1986).

Las asunciones básicas del modelo son las siguientes (Ho y Lee, 1986):

- El mercado no presenta fricciones. No existen costos de transacción ni impuestos, además todos los activos son perfectamente divisibles.

- El mercado se vacía en determinados momentos, separados en intervalos regulares y cada uno de estos periodos se conoce como la unidad de tiempo.

- Se define el bono de descuento de madurez T como aquel que paga \$1 al final del periodo $\mathrm{T}$, sin otros pagos adicionales al tenedor.

- El mercado de bonos es completo.

- Para cada momento $n$ existe un número finito de estados de la naturaleza $i$. Para cada estado existe un precio de equilibrio para el bono de descuento con madurez $T$, dado por $P_{i}^{(n)}(T)$.

- Existe una función estrictamente positiva $P_{i}^{(n)}($.$) , llamada función de descuento$ (FD), y que relaciona el precio de un bono al descuento con su vencimiento; bajo el contexto del modelo esta explica completamente la ETTI en el estado $i$ del momento $n$. Dicha función corresponde a la función de descuento forward implícita para descartar la existencia de oportunidades de arbitraje. Las condiciones que debe satisfacer la FD son: 


$$
P_{i}^{(n)}(0)=1 \forall i, n
$$

La ecuación 5 indica que un bono al descuento que vence instantáneamente debe tener un valor de $\$ 1$.

$$
\lim _{T \rightarrow \infty} P_{i}^{(n)}(T)=0 \forall i, n
$$

La ecuación 6 indica que un bono con un vencimiento lejano debe tener un valor insignificante. Estas asunciones corresponden a las asunciones estándares que garantizan que un mercado de capitales sea perfecto bajo un marco de tiempo discreto.

\subsubsection{El árbol binomial}

En esta sección se describe la evolución de la ETTI, en el momento inicial se tiene que la FD es la siguiente:

$$
P(.)=P_{0}{ }^{(0)}(.)
$$

En cada momento, la FD solo podrá pertenecer a un estado (al alza o a la baja) y en el siguiente momento, esta se convertirá en una FD que puede corresponder a un estado al alza o a la baja. Existen probabilidades neutrales al riesgo que describen la transición de un nodo al siguiente (figura 2). Los nodos de cada momento se relacionan entre sí a través de un árbol binomial, que forma la grilla utilizada para representar la ETTI. Tal diagrama se compone de $M$ pasos y, por tanto, los intervalos temporales están definidos por la siguiente expresión:

$$
\Delta t=\frac{T}{M}
$$

En la figura 2, la posición de cada vértice del árbol binomial puede describirse por $(n, i)$, donde para cada momento del tiempo $n$ existen $(n+1)$ estados $(i=$ $0,1, \ldots, n)$. 
Figura 2: Función de descuento definida para cada momento $n$ y estado $i$

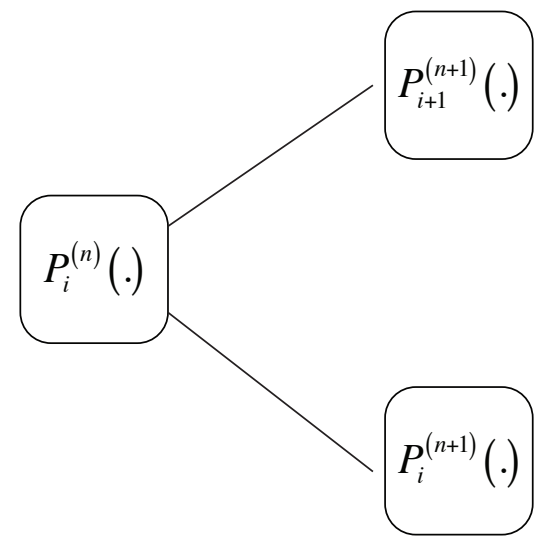

Para derivar el modelo es preciso desarrollar tres etapas:

- Definir la función de perturbación.

- Determinar las probabilidades neutrales al riesgo.

- Establecer la condición de path-independence en el árbol binomial.

\subsubsection{La función de perturbación}

Estas funciones definen las desviaciones de la FD. Es decir, especifican la diferencia entre el estado al alza y a la baja en los precios que rigen durante el próximo periodo.

Bajo un escenario sin incertidumbre, donde no se percibe interés en el próximo periodo, el valor de los dos estados debe ser el mismo, y para evitar la aparición de oportunidades de arbitraje la función de descuento debe corresponder a la función forward implícita $F_{i}^{(n)}(T)$, así se tiene que:

$$
F_{i}^{(n)}(T)=P_{i}^{(n+1)}(T)=P_{i+1}^{(n+1)}(T)=\frac{P_{i}^{(n)}(T+1)}{P_{i}^{(n)}(1)} \quad \text { donde } T=0,1, \ldots
$$

Sin embargo, al modelar la ETTI con incertidumbre la clave consiste en conocer cómo la función de descuento varía el próximo periodo de acuerdo con la función forward implícita. Para esto se definen las funciones de perturbación, $h(T)$ y $h^{*}(T)$. 
En el estado al alza:

$$
P_{i+1}^{(n+1)}(T)=\frac{P_{i}^{(n)}(T+1)}{P_{i}^{(n)}(1)} h(T)
$$

En el estado a la baja:

$$
P_{i+1}^{(n+1)}(T)=\frac{P_{i}^{(n)}(T+1)}{P_{i}^{(n)}(1)} h^{*}(T)
$$

Las funciones de perturbación especifican las desviaciones de las funciones de descuento respecto a la función forward implícita, es decir, especifican las diferencias entre los estados al alza y a la baja en el próximo periodo. Para cumplir con las ecuaciones 5 y 6 es preciso que se cumpla:

$$
h(0)=h^{*}(0)=1
$$

\subsubsection{Probabilidad binomial implícita}

Para evitar la aplicación de estrategias de arbitraje se establecen restricciones a las funciones de perturbación en cada vértice del árbol. El resultado muestra que si el movimiento al alza es muy grande, el movimiento a la baja debe ser similar, de forma que el promedio ponderado de todos los movimientos sea el mismo para todos los bonos. Ho y Lee demuestran que la restricción es la siguiente:

$$
\pi^{*} h(T)+(1-\pi) * h^{*}(\mathrm{~T})=1 \text { para } \forall n, i>0
$$

Esta probabilidad implícita es la equivalente a la probabilidad neutral al riesgo en el contexto de Cox, Ross y Rubinstein, y puede ser reescrita de la siguiente forma:

$$
\begin{aligned}
& P_{i}^{(n)}(T)=\left[\pi P_{i+1}^{(n+1)}(T-1)+(1-\pi) P_{i}^{(n+1)}(T-1)\right] P_{i}^{(n)}(1) \\
& \pi=\frac{r-d}{u-d}
\end{aligned}
$$


donde:

$r$ : retorno del bono para un periodo.

$u$ y $d$ : los retornos del bono en los estados al alza y a la baja, respectivamente.

Por tanto, la probabilidad $\pi$ es una medida del retorno en el estado a la baja respecto del spread entre los dos estados. Es decir, si la probabilidad es alta, los cambios en los precios estarán dominados por descensos en los precios y viceversa (ver ecuación 14).

\subsubsection{Condición de path-independence}

La condición de path-independence exige que la función de descuento debe evolucionar de un estado al otro de acuerdo con el número de estados al alza y a la baja, independientemente del orden en que se presenten. Por tanto, se debe imponer una restricción a las funciones de perturbación como a la probabilidad binomial implícita de forma que un movimiento al alza, seguido por uno a la baja, produzcan uno equivalente a un movimiento a la baja seguido por un movimiento al alza. A continuación se utilizan las ecuaciones 10 y 11 para representar esta situación:

$$
P_{i+1}^{(n+2)}(T)=\frac{P_{i}^{(n)}(T+2)}{P_{i}^{(n)}(2)} \frac{h(T+1) h^{*}(T)}{h(1)}=\frac{P_{i}^{(n)}(T+2)}{P_{i}^{(n)}(2)} \frac{h^{*}(T+1) h(T)}{h^{*}(1)}
$$

Simplificando la ecuación 15 se obtiene:

$$
h(T+1) h^{*}(T) h^{*}(T+1) h(T) h(1)
$$

Se utiliza la ecuación 13 para dejar la expresión anterior en términos de la probabilidad implícita y $\mathrm{h}(\mathrm{T})$ :

$$
h(T+1)[1-\pi h(T)][1-\pi h(1)]=(1-\pi) h(1) h(T)[1-\pi h(T+1)]
$$


De lo anterior se obtiene:

$$
\frac{1}{h(T+1)}=\frac{\delta}{h(T)}+\gamma
$$

La constante $\delta$ debe satisfacer:

$$
h(1)=\frac{1}{(\pi+(1-\pi)) \delta}
$$

$\mathrm{y}$

$$
\gamma=\frac{\pi(h(1)-1)}{(1-\pi) h(1)}
$$

La solución de la ecuación diferencial de primer orden (ecuación 18):

$$
\begin{gathered}
h(T)=\frac{1}{\pi+(1-\pi) \delta^{T}} \quad \forall T \geq 0 \\
h^{*}(T)=\frac{\delta^{T}}{\pi+(1-\pi) \delta^{T}}
\end{gathered}
$$

\subsubsection{Modelo de tasas libre de arbitraje}

De acuerdo con Ho y Lee (1986), la expresión explícita de la función de descuento en cualquier estado y tiempo es:

$$
P_{i}^{(n)}(T)=\frac{P(T+n) h(T+n-1) h(T+n-2) . . . h(T) \delta^{T(n-1)}}{P(n) h(n-1) h(n-2) . . h(1)}
$$


Por tanto, la tasa de interés de un periodo será:

$$
r_{i}^{(n)}=-\operatorname{In} P_{i}^{(n)}(1)=\operatorname{In}\left[\frac{P(n)}{P(n+1)}\right]+\operatorname{In}\left(\pi \delta^{-n}+(1-\pi)\right)+\mathrm{i} \ln \delta
$$

A diferencia del caso binomial usual, en el que se calcula la probabilidad neutral al riesgo a partir de los estados al alza $(u)$ y a la baja $(d)$, en este trabajo se utilizó el modelo calibrado a partir de la definición de la amplitud de los movimientos fijando la probabilidad neutral al riesgo en 0,5 , siguiendo el procedimiento establecido en Ho y Lee (2004) y van Straaten (2009). Cuando el modelo se calibra utilizando probabilidades neutrales al riesgo de 0,5 , la expresión para la tasa de interés de un periodo se calcula con la siguiente expresión (Ho y Lee, 2004):

$$
r_{i}^{(n)}(1)=\operatorname{In}\left[\frac{P(n)}{P(n+1)}\right]+\operatorname{In}\left(0.5\left(\delta^{-\frac{n}{2}}+\delta^{\frac{n}{2}}\right)\right)+\left(\frac{n}{2}-i\right) \operatorname{In} \delta
$$

Y la expresión para hallar los factores de descuento con probabilidades neutrales al riesgo de 0,5 , que se utilizan para calcular el precio de cada bono entregable en la fecha del delivery, es la siguiente (van Straaten, 2009):

$$
H\left((M, s), t_{i}^{j}\right)=\frac{P\left(0, t_{i}^{j}\right)}{P\left(0, t_{d}\right)} e^{-\left(t_{i}^{j}-t_{d}\right)\left(\hat{r}(M, s)-f\left(0, t_{d}\right)\right)-\frac{1}{2} \sigma^{2} t_{d}\left(t_{i}^{j}-t_{d}\right)^{2}}
$$

\section{Resultados}

\subsection{Descripción de los datos}

Los datos utilizados durante la realización del estudio corresponden a:

- La serie de precios de bonos de deuda pública emitidos a tasa fija en pesos para el periodo entre septiembre de 2008 y 2013. Los precios corresponden a las transacciones en el Sistema Electrónico de Negociación del Banco de la República de Colombia, que se publican diariamente y pueden consultarse en la página web de esta institución. 
- Las tasas de la curva swap del mercado monetario colombiano, Overnight Index Swap (OIS), para el corto plazo. A pesar de que la consulta se realizó en Bloomberg desde 2008, no todos los plazos presentan información desde el inicio de la serie, debido al nivel de desarrollo del mercado de swaps de tasas de interés colombiano.

- Los datos de la figura 2 se obtuvieron del aplicativo de la CRCC.

Información general sobre el futuro por analizar.

Tabla 2: Características del futuro por analizar

\begin{tabular}{|c|c|}
\hline Futuro & TEMH12F \\
\hline Fecha de vencimiento & $03 / 02 / 2012$ \\
\hline Fecha de primera negociación & $28 / 11 / 2011$ \\
\hline Fecha de última negociación & $29 / 02 / 2012$ \\
\hline Fecha de interés & $29 / 11 / 2011$ \\
\hline
\end{tabular}

\begin{tabular}{|l|c|}
\hline \multicolumn{2}{|c|}{ Factores de conversión de entregables } \\
\hline 1. TFIT10281015 & 0,91 \\
\hline 2. TFIT07150616 & 0,88 \\
\hline 3. TFIT11241018 & 1,01 \\
\hline
\end{tabular}

Fuente: elaboración propia.

Con el objeto de identificar si el CTD cambia al variar las tasas, se calculó el precio del futuro en la fecha actual para diferentes niveles de tasa. En la figura 3 se observa que el entregable TFIT07150616 ${ }^{6}$ nunca se convierte en el CTD, mientras que el TFIT10281015 es el CTD cuando la tasa disminuye y el TFIT11241018 es el CTD cuando la tasa aumenta.

6 El mnemotécnico de los títulos de deuda pública se compone de los siguientes elementos TFIT(TES tasa fija en pesos)+ 07 (periodo inicial al vencimiento del título) + ddmmaa (fecha de vencimiento del título). 
Figura 3: Precio del futuro vs. movimientos en las tasas para el contrato TEMH12F

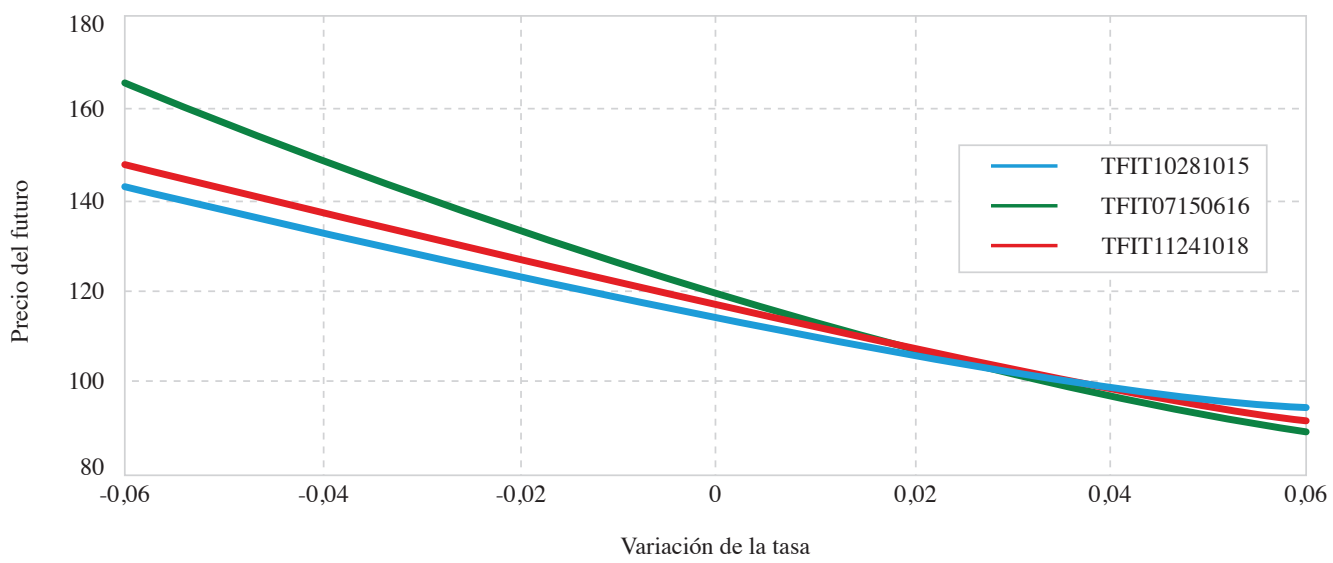

\subsection{Cálculo de la curva cero cupón}

Para calcular la CCC se utilizó la metodología de bootstrapping, usando la función de MatLab zbtprice (Anexo 1).

Tabla 3: Información para construir la curva cero cupón

\begin{tabular}{|l|c|}
\hline \multicolumn{2}{|c|}{ Factores de conversión de entregables } \\
\hline IBR overnight & 0,04820 \\
\hline IBR 30 días & 0,04818 \\
\hline
\end{tabular}

\begin{tabular}{|c|c|c|c|c|}
\hline Títulos del mercado & Precios de cierrre & Cupón & Fecha de vencimiento & Días al vencimiento \\
\hline TFIT04150812 & 102,532 & 9,25 & $2012-08-15$ & 260 \\
\hline TFIT04170413 & 100,157 & 6 & $2013-04-17$ & 505 \\
\hline TFIT06140514 & 106,566 & 9,25 & $2014-05-14$ & 897 \\
\hline TFIT10281015 & 104,499 & 8 & $2015-10-28$ & 1429 \\
\hline TFIT07150616 & 101,753 & 7,25 & $2016-06-15$ & 1660 \\
\hline TFIT11241018 & 120,957 & 11,25 & $2018-10-24$ & 2521 \\
\hline TFIT16240724 & 118,330 & 10 & $2024-07-24$ & 4621 \\
\hline
\end{tabular}

Fuente: elaboración propia. 
La curva cero cupón calculada en este caso tiene la siguiente forma:

Tabla 4: Curva cero cupón contrato TEMH12F

\begin{tabular}{|c|c|c|c|}
\hline Días al vencimiento & Años & Fecha de vencimiento & Tasa \\
\hline 1 & 0,002739726 & $30 / 11 / 11$ & 0,0483293413 \\
\hline 30 & 0,082191781 & $29 / 12 / 11$ & 0,0483320548 \\
\hline 260 & 0,712328767 & $15 / 08 / 12$ & 0,0528144350 \\
\hline 505 & 1,383561644 & $17 / 04 / 13$ & 0,0568575777 \\
\hline 897 & 2,457534247 & $14 / 05 / 14$ & 0,0608732897 \\
\hline 1429 & 3,915068493 & $28 / 10 / 15$ & 0,0649600410 \\
\hline 1660 & 4,547945205 & $15 / 06 / 16$ & 0,0661726695 \\
\hline 2521 & 6,906849315 & $24 / 10 / 18$ & 0,0721659815 \\
\hline 4621 & 12,66027397 & $24 / 07 / 24$ & 0,0764694028 \\
\hline
\end{tabular}

Fuente: elaboración propia.

Figura 4: Curva cero cupón - Contrato TEMH12F

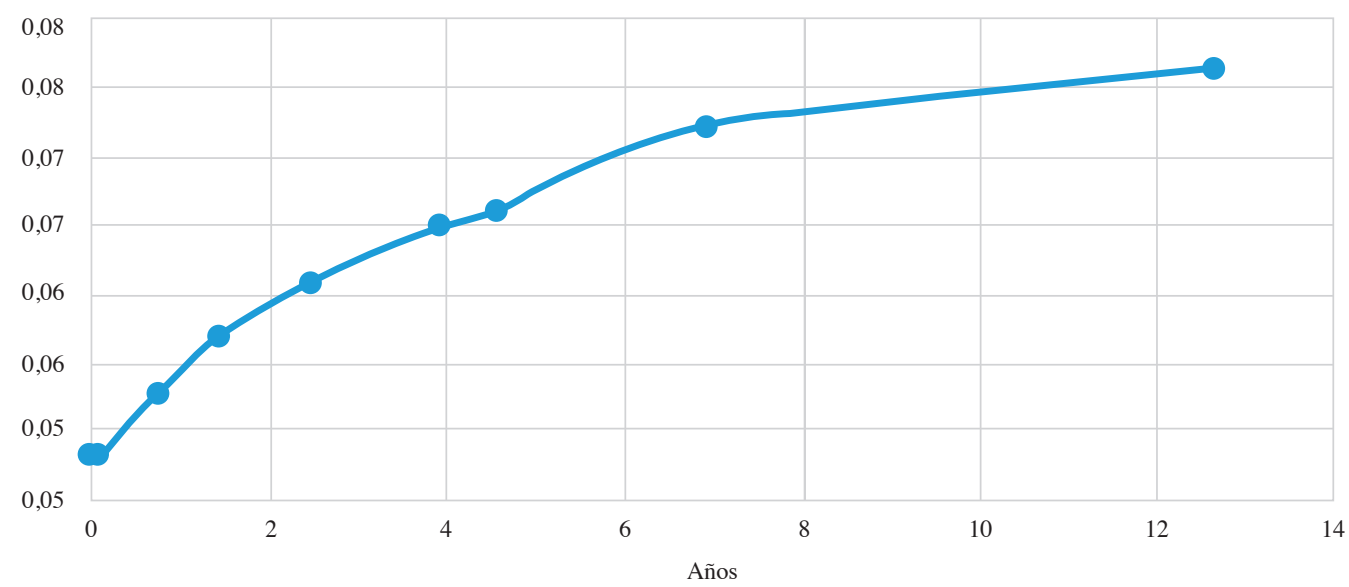

En la construcción de la curva cero cupón se incluyeron las tasas del mercado OIS para los plazos de 1 y 30 días, con el fin de tener una referencia de corto plazo que permitiera realizar interpolación para hallar la tasa del primer paso del árbol Ho-Lee. Las tasas de este mercado pueden considerarse comparables con las provenientes de los títulos de deuda pública, porque se asume que son productos con bajo riesgo crediticio. 


\section{3. Árbol de Ho-Lee}

En el proceso de construcción del árbol se utiliza la expresión de la ecuación 25 presentada por Ho y Lee (2004, pp. 139 y 140):

$$
r_{i}^{(n)}(1)=\operatorname{In}\left[\frac{P(n)}{P(n+1)}\right]+\operatorname{In}\left(0.5\left(\delta^{-\frac{n}{2}}+\delta^{\frac{n}{2}}\right)\right)+\left(\frac{n}{2}-i\right) \operatorname{In} \delta
$$

donde:

El primer término corresponde a la tasa forward a un periodo.

El segundo término corresponde al ajuste por la convexidad característica de los bonos.

El tercer término corresponde a movimientos ascendentes y descendentes de la curva luego de $n$ periodos (movimientos independientes del nivel de la tasa). $n$ : número de pasos del árbol.

I: ubicación en el árbol en cada paso.

$P(n)$ : precio del bono cero cupón en el momento «n».

$\sigma$ : volatilidad de la tasa de interés de corto plazo.

$$
\begin{aligned}
& P_{i}^{(n)}(1)=2\left[\frac{P(n+1)}{P(n)}\right] * \frac{\delta^{i}}{\left(1+\delta^{n}\right)} \\
& P(n)=e^{(-n * z(n))} \\
& \delta=e^{-2 r(1) \sigma}
\end{aligned}
$$

Tabla 5: Información para la construcción del árbol de Ho-Lee

\begin{tabular}{|c|c|}
\hline Número de días desde la fecha actual hasta la fecha del delivery & 92 \\
\hline Número de pasos del árbol & 4 \\
\hline Número de días de cada periodo & 23 \\
\hline Volatilidad & 0,009146 \\
\hline Delta & 0,999116309 \\
\hline
\end{tabular}

Fuente: elaboración propia. 
La volatilidad de la tasa de interés de corto plazo corresponde a la máxima desviación estándar de la serie histórica de las tasas de retorno de los títulos entregables del contrato en cuestión, calculada como:

$$
\sigma=\left[\text { desviación estándar }\left(r_{\text {hoy }}-r_{\text {ayer }}\right)\right] * \sqrt{\text { Tamaño del paso }}
$$

Con base en la CCC, y utilizando el método de interpolación log linear on the discount factors (ecuación 3), se calculan las tasas cero cupón para los respectivos nodos del árbol (tabla 6):

Tabla 6: Cálculos intermedios en la construcción del árbol de tasas de Ho-Lee

\begin{tabular}{|c|c|c|}
\hline Pasos & Días & Tasas cero cupón \\
\hline 1 & 23,000 & 0,04833 \\
\hline 2 & 46,000 & 0,05009 \\
\hline 3 & 69,000 & 0,05120 \\
\hline 4 & 92,000 & 0,05175 \\
\hline 5 & 115,00 & 0,05208 \\
\hline
\end{tabular}

\begin{tabular}{|c|c|}
\hline Pasos & Precio del bono P(n) \\
\hline 1 & 0,99695905 \\
\hline 2 & 0,99370661 \\
\hline 3 & 0,99036853 \\
\hline 4 & 0,98704166 \\
\hline 5 & 0,98372597 \\
\hline
\end{tabular}

Fuente: elaboración propia.

Tabla 7: Árbol de tasas por medio de la metodología Ho-Lee

\begin{tabular}{|c|c|c|c|c|c|c|c|c|}
\hline Hoy & & 1 & & 2 & & 3 & & 4 \\
\hline \multirow[t]{5}{*}{$4,83 \%$} & 1 & $5,89 \%$ & 2 & $6,74 \%$ & 3 & $7,45 \%$ & 4 & $8,15 \%$ \\
\hline & 0 & $4,48 \%$ & 1 & $5,34 \%$ & 2 & $6,04 \%$ & 3 & $6,75 \%$ \\
\hline & & & 0 & $3,94 \%$ & 1 & $4,64 \%$ & 2 & $5,34 \%$ \\
\hline & & & & & 0 & $3,24 \%$ & 1 & $3,94 \%$ \\
\hline & & & & & & & 0 & $2,54 \%$ \\
\hline
\end{tabular}

Fuente: elaboración propia. 


\subsection{Cálculo del cheapest to deliver}

La ecuación (1) presenta la relación entre el CTD, el precio del bono, el factor de conversión y el precio del contrato de futuro. Para hallar el CTD se debe hallar el precio del bono y el de los contratos de futuros en la fecha del delivery utilizando la CCC y las tasas del árbol de Ho-Lee encontrados en las dos secciones anteriores.

\subsubsection{Cálculo del precio de los bonos en la fecha de delivery}

El precio de los bonos se calcula a partir del descuento de los flujos futuros y pago final, en la fecha del delivery. Para ello se utiliza la siguiente expresión:

$$
K\left(\vec{c}^{j}, \vec{t}_{c}^{j},(M, s)\right)=\sum_{i=1}^{N} c_{t_{i}}^{j} H\left((M, s), t_{i}^{j}\right)+100 H\left((M, s), t_{N}^{j}\right)
$$

donde:

$\vec{c}^{j}$ : cupones

$\vec{t}_{c}^{j}$ :momento en el que se pagan los cupones

$(M, s)$ : posición en el árbol de $\mathrm{M}$ pasos

$H\left((M, s), t_{i}^{j}\right):$ factor de descuento

El factor de descuento se halla a partir de la siguiente expresión (ecuación 26):

$$
H\left((M, s), t_{i}^{j}\right)=\frac{P\left(0, t_{i}^{j}\right)}{P\left(0, t_{d}\right)} e^{-\left(t_{i}^{j}-t_{d}\right)\left(\hat{r}(M, s)-f\left(0, t_{d}\right)\right)-\frac{1}{2} \sigma^{2} t_{d}\left(t_{i}^{j}-t_{d}\right)^{2}}
$$

donde:

$$
\begin{aligned}
& P(t, T)=e^{-(T-t) z(t, T)} \\
& f(t, T)=\frac{\partial}{\partial T} \operatorname{In} P(t, T)
\end{aligned}
$$


La tasa forward entre los puntos de interés es constante y corresponde a la expresión presentada en la ecuación 4 . A continuación se muestran los resultados de los factores de descuento para cada flujo de los títulos entregables y los precios de los mismos en los nodos del último paso del árbol.

Tabla 8: Factores de descuento para cada flujo y posición en el árbol - Contrato TEMH12F

\begin{tabular}{|c|c|c|c|c|c|c|}
\hline \multicolumn{7}{|c|}{ Factores de descuento } \\
\hline & & \multicolumn{5}{|c|}{ Posición en el árbol } \\
\hline & Flujos & $(4,4)$ & $(3,4)$ & $(2,4)$ & $(1,4)$ & $(0,4)$ \\
\hline \multirow[t]{6}{*}{ TFIT10281015 } & 1 & 0,94502042 & 0,95385208 & 0,96276628 & 0,97176379 & 0,98084538 \\
\hline & 2 & 0,86068685 & 0,88100455 & 0,90180189 & 0,92309017 & 0,94488099 \\
\hline & 3 & 0,77999183 & 0,80968517 & 0,8405089 & 0,87250606 & 0,90572131 \\
\hline & 4 & 0,70462557 & 0,74178437 & 0,78090276 & 0,82208408 & 0,86543712 \\
\hline & & \multicolumn{5}{|c|}{ Posición en el árbol } \\
\hline & Flujos & $(4,4)$ & $(3,4)$ & $(2,4)$ & $(1,4)$ & $(0,4)$ \\
\hline \multirow[t]{6}{*}{ TFIT07150616 } & 1 & 0,97605255 & 0,98007523 & 0,98411448 & 0,98817038 & 0,992243 \\
\hline & 2 & 0,89172269 & 0,90804877 & 0,92467376 & 0,94160312 & 0,95884244 \\
\hline & 3 & 0,80986084 & 0,83634007 & 0,86368506 & 0,89192412 & 0,92108649 \\
\hline & 4 & 0,73161421 & 0,76620994 & 0,8024416 & 0,84038653 & 0,88012576 \\
\hline & 5 & 0,65996754 & 0,7009678 & 0,74451518 & 0,79076793 & 0,83989411 \\
\hline & & \multicolumn{5}{|c|}{ Posición en el árbol } \\
\hline \multirow[t]{8}{*}{ TFIT11241018 } & Flujos & $(4,4)$ & $(3,4)$ & $(2,4)$ & $(1,4)$ & $(0,4)$ \\
\hline & 1 & 0,94596188 & 0,95465555 & 0,96342912 & 0,97228332 & 0,98121889 \\
\hline & 2 & 0,86159075 & 0,8817942 & 0,90247141 & 0,92363347 & 0,94529177 \\
\hline & 3 & 0,78086083 & 0,81046263 & 0,84118662 & 0,87307532 & 0,9061729 \\
\hline & 4 & 0,70541077 & 0,74249681 & 0,78153259 & 0,82262064 & 0,86586883 \\
\hline & 5 & 0,63362018 & 0,67638091 & 0,72202742 & 0,77075444 & 0,82276987 \\
\hline & 6 & 0,56561885 & 0,6123213 & 0,66287992 & 0,71761309 & 0,77686552 \\
\hline & 7 & 0,50490491 & 0,55431705 & 0,60856487 & 0,6681216 & 0,73350682 \\
\hline
\end{tabular}

Fuente: elaboración propia. 
A partir de los factores de descuento de la tabla 8 es posible calcular las tasas equivalentes y con ellas construir una curva de tasas para cada nodo final del árbol. Estas curvas se extienden desde la fecha del delivery hasta la fecha del último flujo de los entregables. Dichas tasas se calcularon usando la siguiente ecuación:

$$
r\left((M, s), t_{i}^{j}\right)=-\frac{\operatorname{In} H\left((M, s), t_{i}^{j}\right)}{\left(t_{i}^{j}-t_{d}\right)}
$$

Tabla 9: Árbol de tasas Ho-Lee - Contrato TEMH12F
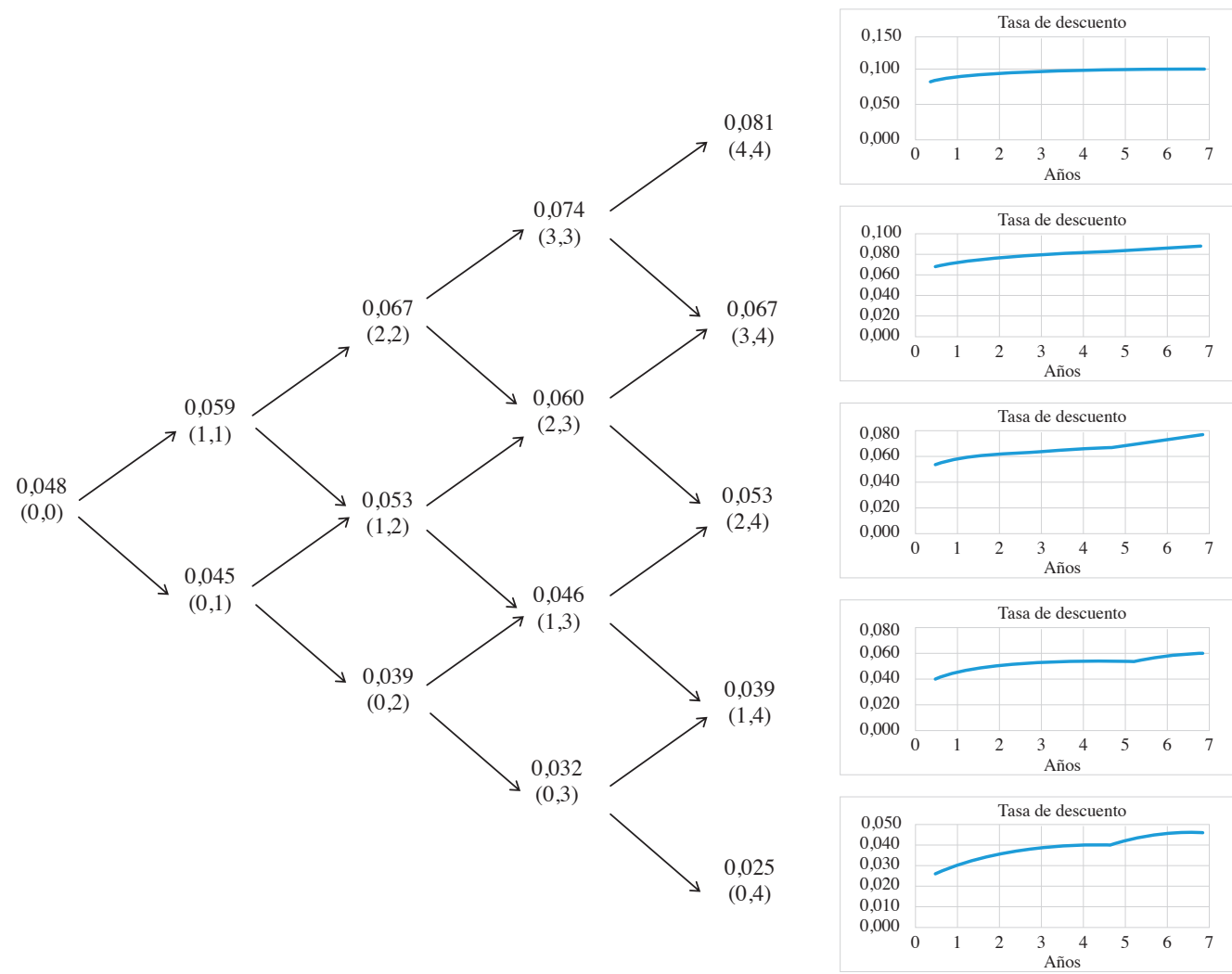

Fuente: elaboración propia.

El precio limpio de los bonos entregables se calcula como la sumatoria de los valores resultantes al multiplicar cada factor de descuento hallado en la tabla 8 por el valor del respectivo flujo (cupón o valor facial), y luego se le resta el valor de los intereses devengados hasta la fecha. 
Tabla 10: Precio de los bonos entregables en la fecha del delivery - Contrato TEMH12F

Precio de los bonos en la fecha del delivery

\begin{tabular}{|c|c|}
\hline Fecha actual & $29 / 11 / 11$ \\
\hline Última fecha que pagó cupón & $14 / 05 / 11$ \\
\hline Cupón & 8 \\
\hline Número de días & 199 \\
\hline Intereses devengados & 4,361643836 \\
\hline
\end{tabular}

\begin{tabular}{|c|c|c|c|c|c|c|}
\hline \multicolumn{2}{|c|}{} & \multicolumn{6}{|c|}{ Posición en el árbol } \\
\cline { 2 - 8 } & Flujos & $(4,4)$ & $(3,4)$ & $(2,4)$ & $(1,4)$ & $(0,4)$ \\
\hline \multirow{2}{*}{ TFIT10281015 } & 1 & 7,56016334 & 7,63081665 & 7,70213024 & 7,7741103 & 7,84676304 \\
\hline & 2 & 6,88549477 & 7,04803642 & 7,21441508 & 7,38472135 & 7,55904792 \\
\cline { 2 - 8 } & 3 & 6,23993462 & 6,47748137 & 6,72407123 & 6,98004848 & 7,24577046 \\
\cline { 2 - 8 } & 4 & 76,0995616 & 80,1127122 & 84,3374983 & 88,7850808 & 93,4672089 \\
\hline Precio Sucio & & 96,78515 & 101,26905 & 105,97811 & 110,92396 & 116,11879 \\
\hline Precio Limpio & & 92,42351 & 96,90740 & 101,61647 & 106,56232 & 111,75715 \\
\hline
\end{tabular}

\begin{tabular}{|c|c|}
\hline Fecha actual & $29 / 11 / 11$ \\
\hline Última fecha que pagó cupón & $15 / 06 / 11$ \\
\hline Cupón & 7,25 \\
\hline Número de días & 167 \\
\hline Intereses devengados & 3,317123288 \\
\hline
\end{tabular}

\begin{tabular}{|c|c|c|c|c|c|c|}
\hline & \multicolumn{6}{|c|}{ Posición en el árbol } \\
\cline { 2 - 8 } & Flujos & $(4,4)$ & $(3,4)$ & $(2,4)$ & $(1,4)$ & $(0,4)$ \\
\hline \multirow{2}{*}{ TFIT07150616 } & 1 & 7,07638101 & 7,1055454 & 7,13482999 & 7,16423526 & 7,19376173 \\
\hline \multirow{5}{*}{} & 2 & 9,69289589 & 9,92018475 & 10,1528033 & 10,3908765 & 10,6345324 \\
\cline { 2 - 8 } & 3 & 8,7846843 & 9,1177046 & 9,46334943 & 9,82209738 & 10,1944452 \\
\cline { 2 - 8 } & 4 & 7,93587112 & 8,35308906 & 8,79224168 & 9,25448217 & 9,74102434 \\
\cline { 2 - 8 } & 5 & 7,12822698 & 7,60928528 & 8,12280846 & 8,67098746 & 9,25616108 \\
\cline { 2 - 8 } & 6 & 6,36321208 & 6,88861465 & 7,45739905 & 8,07314729 & 8,73973709 \\
\cline { 2 - 8 } & 7 & 56,1706716 & 61,6677717 & 67,7028413 & 74,3285284 & 81,6026333 \\
\hline Precio Sucio & & 106,71763 & 114,29652 & 122,53002 & 131,47831 & 141,20725 \\
\hline Precio Limpio & & 95,46763 & 103,04652 & 111,28002 & 120,22831 & 129,95725 \\
\hline
\end{tabular}

Fuente: elaboración propia. 
Para calcular el CTD en cada nodo de la fecha del delivery se toma el mínimo luego de comparar los valores correspondientes a:

$$
\frac{K\left(\vec{c}^{j}, \vec{t}_{c}^{j},(M, s)\right)}{C F_{j}}
$$

Tabla 11: CTD en la fecha del delivery - Contrato TEMH12F

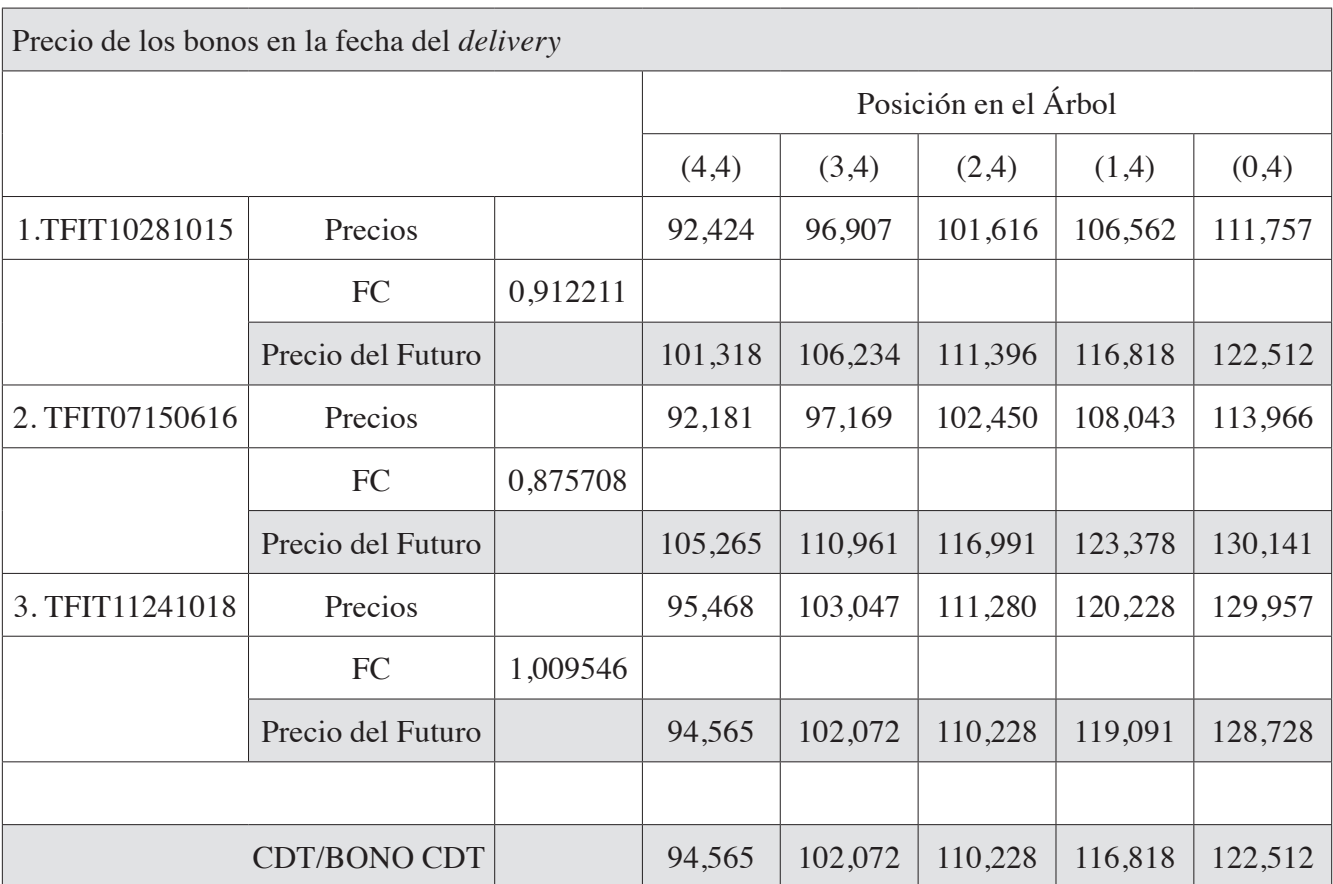

Fuente: elaboración propia.

Dado que las probabilidades neutrales al riesgo corresponden a 0,5, los precios del futuro en los nodos del árbol anteriores a la fecha del delivery se calculan a partir de la siguiente expresión:

$$
F\left((M-1, s), t_{d}\right)=\frac{1}{2}\left[F\left((M, s), t_{d}\right)+F\left((M, s+1), t_{d}\right)\right]
$$




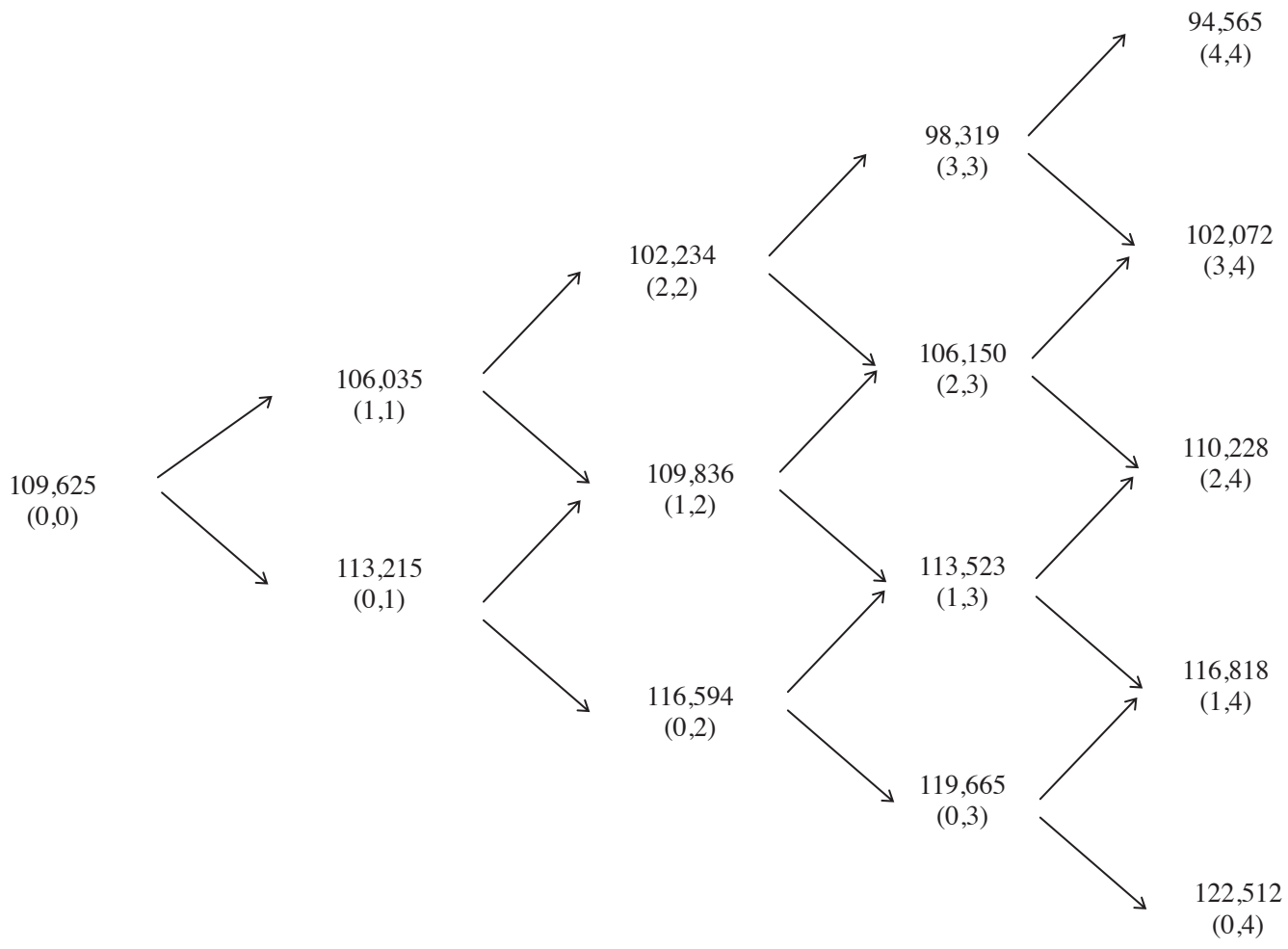

Fuente: elaboración propia.

$\mathrm{Al}$ igual que los precios del futuro, la probabilidad de que un bono se convierta en el CTD en los nodos anteriores al delivery también se halla descontando dicha probabilidad desde los nodos finales hasta el inicio del árbol, usando las probabilidades neutrales al riesgo de 0,5. 
Tabla 13: Árbol de probabilidades del CTD - Contrato TEMH12F

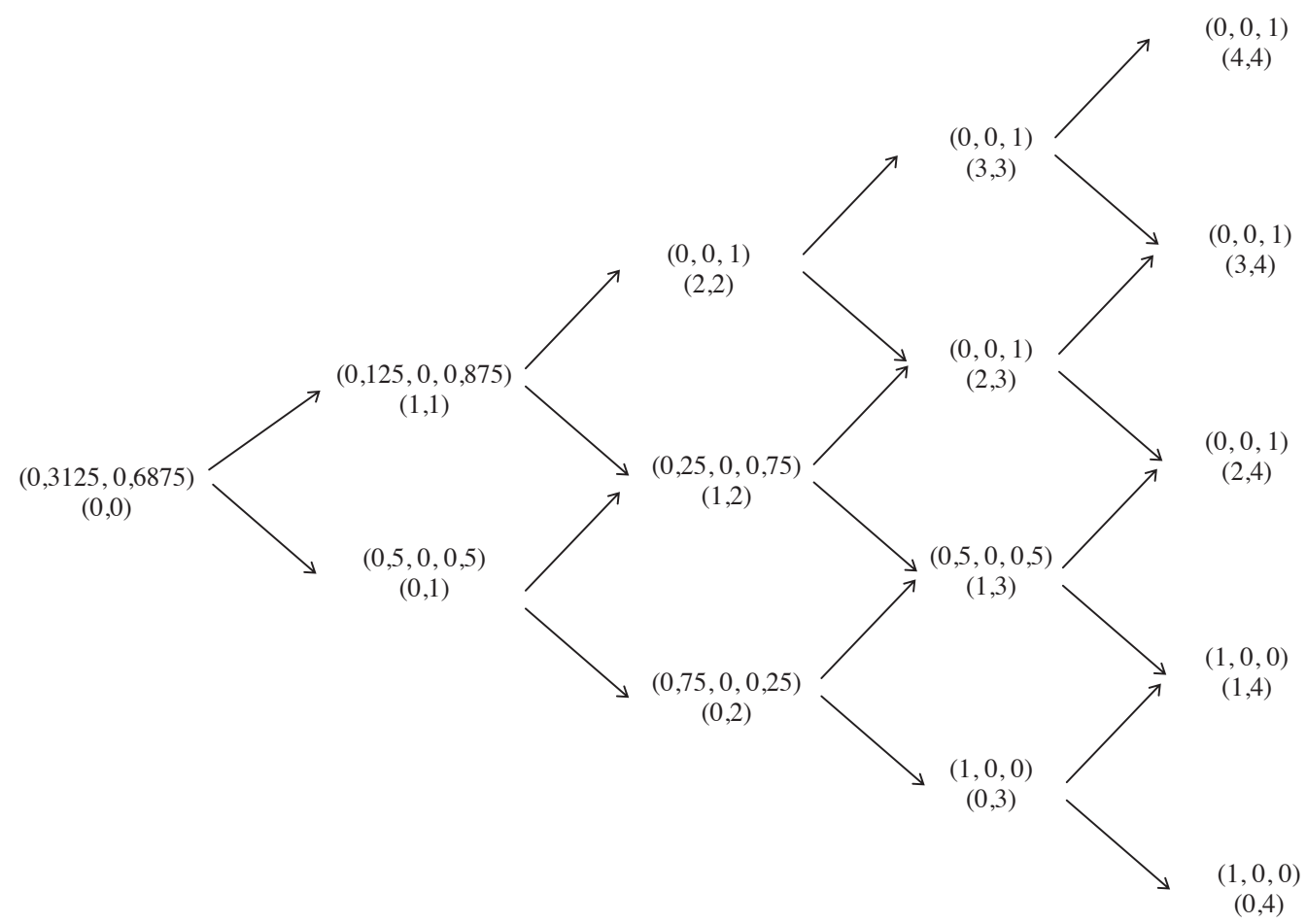

Fuente: elaboración propia.

4.4.1.1. Análisis de sensibilidad TEMH12F: cambios en el CTD vs. cambios en la curva cero cupón

Para determinar los efectos en el CTD de los movimientos de las tasas se analizaron cinco escenarios en los que se modificó el valor de la curva cero cupón (CCC): 1) CCC+100 p.b., 2) CCC-100 p.b., 3) CCC+200 p.b., 4) CCC-200 p.b. у 5) CCC -300 p.b. (figura 5). Si bien se evidencian cambios en el valor del futuro, el CTD en cada nodo es el mismo que resulta con los datos iniciales. Adicionalmente, es importante resaltar que se mantienen las relaciones expuestas en la figura 3:

- El TFIT07150616 nunca se convierte en el CTD.

- El TFIT10281015 es el CTD para tasas más bajas (nodos $[1,4]$ y $[0,4]$ ).

- El TFIT11241018 es el CTD para tasas más altas (nodos [4,4], [3,4] y [2,4]). 
Figura 5: Análisis de sensibilidad del CTD ante cambios en la curva cero cupón - Contrato TEMH12F
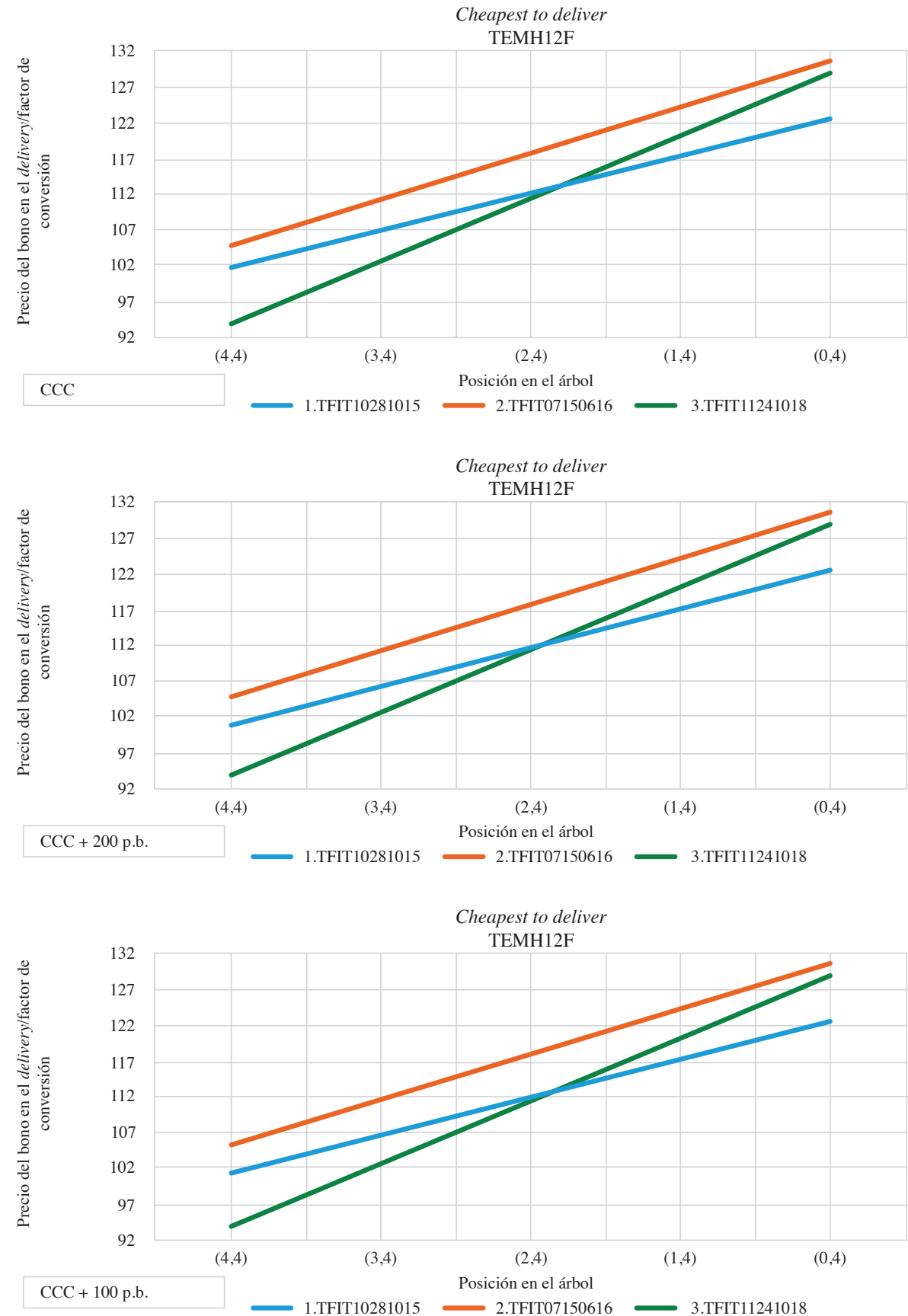

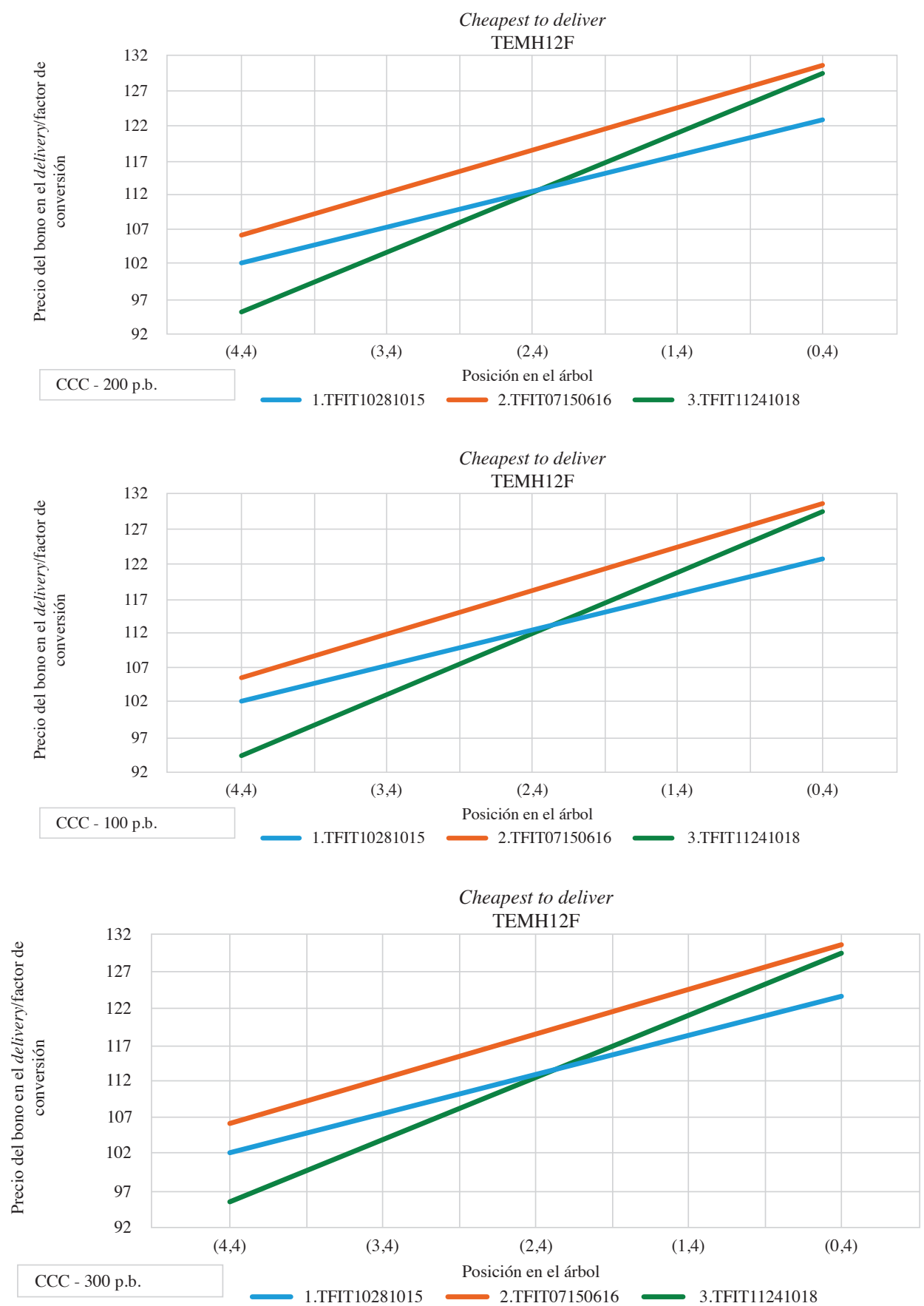
4.4.1.2. Análisis de sensibilidad TEMH12F: cambios en el CTD vs. cambios en la volatilidad

Para determinar los efectos en el CTD de los movimientos de la volatilidad se analizaron seis escenarios en los que se modificó el valor de esta variable: 1) 0,01 ; 2) 0,015 ; 3) 0,02 ; 4) 0,025 ; 5) 0,035 y 6) 0,1 (figura 6). Si bien se evidencian cambios en el valor del futuro, el CTD en cada nodo es el mismo que resulta con los datos iniciales. Sin embargo, es importante resaltar que se mantienen las relaciones expuestas en secciones precedentes:

- El TFIT07150616 nunca se convierte en el CTD.

- El TFIT10281015 es el CTD para tasas más bajas (nodos $[1,4]$ y $[0,4]$ ).

- El TFIT11241018 es el CTD para tasas más altas (nodos $[4,4],[3,4]$ y $[2,4]$ ).

Figura 6: Análisis de sensibilidad del CTD ante cambios en la volatilidad - Contrato TEMH12F

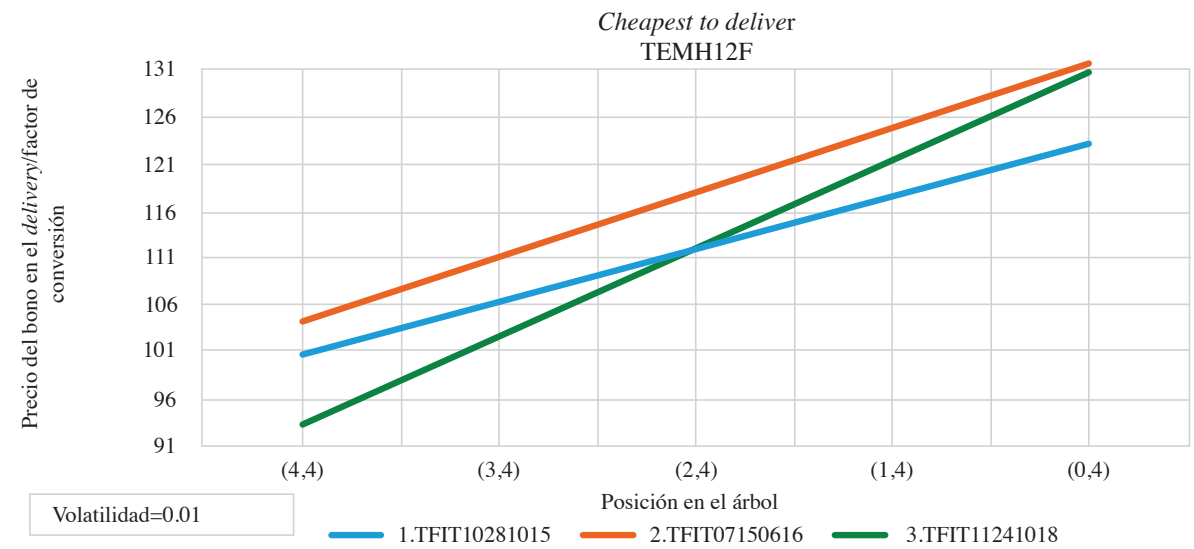



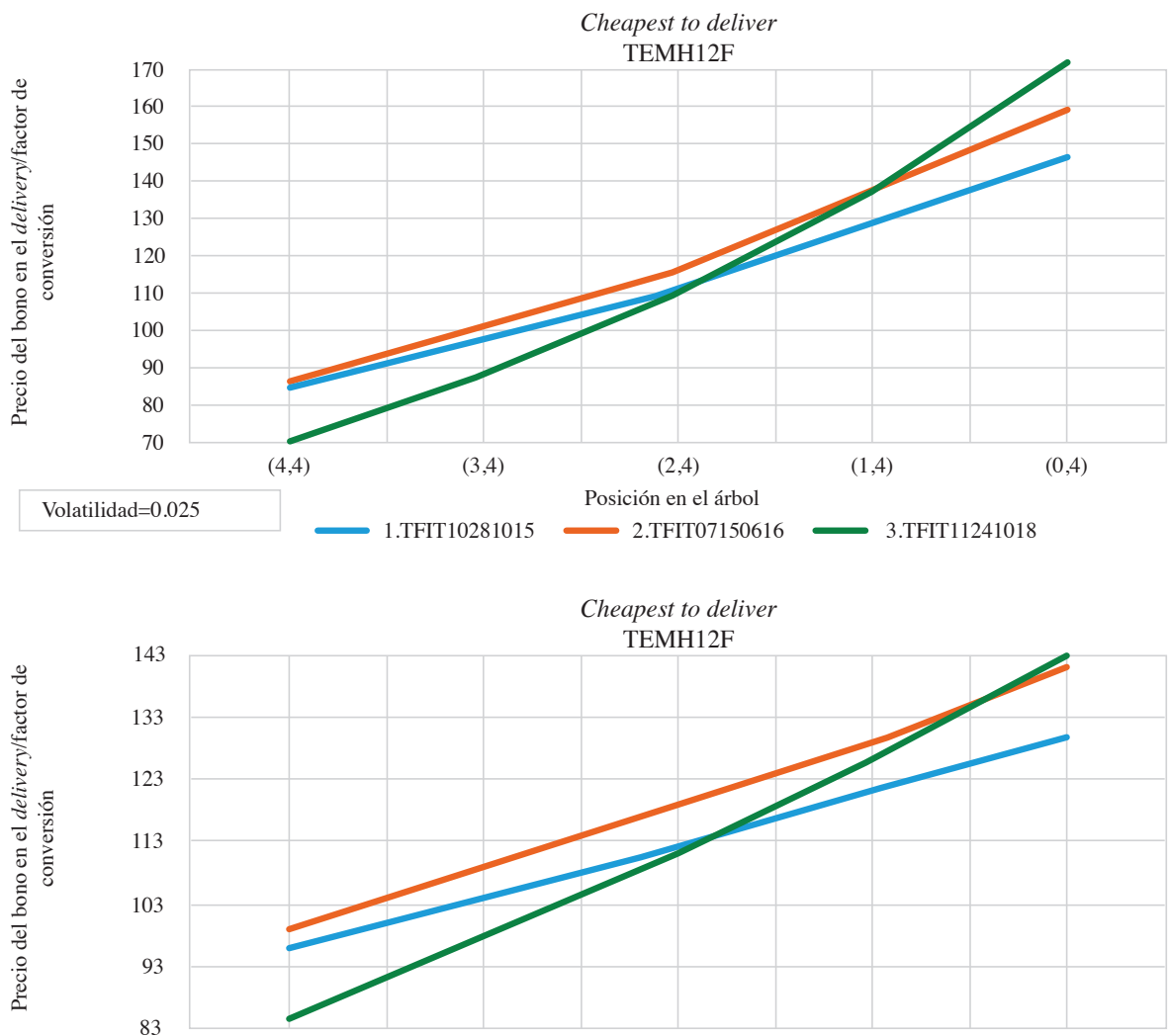

$(4,4)$

$(3,4)$

$(2,4)$

$(1,4)$

$(0,4)$

Volatilidad $=0.015$

Posición en el árbol 1.TFIT10281015 2.TFIT07150616

3.TFIT11241018

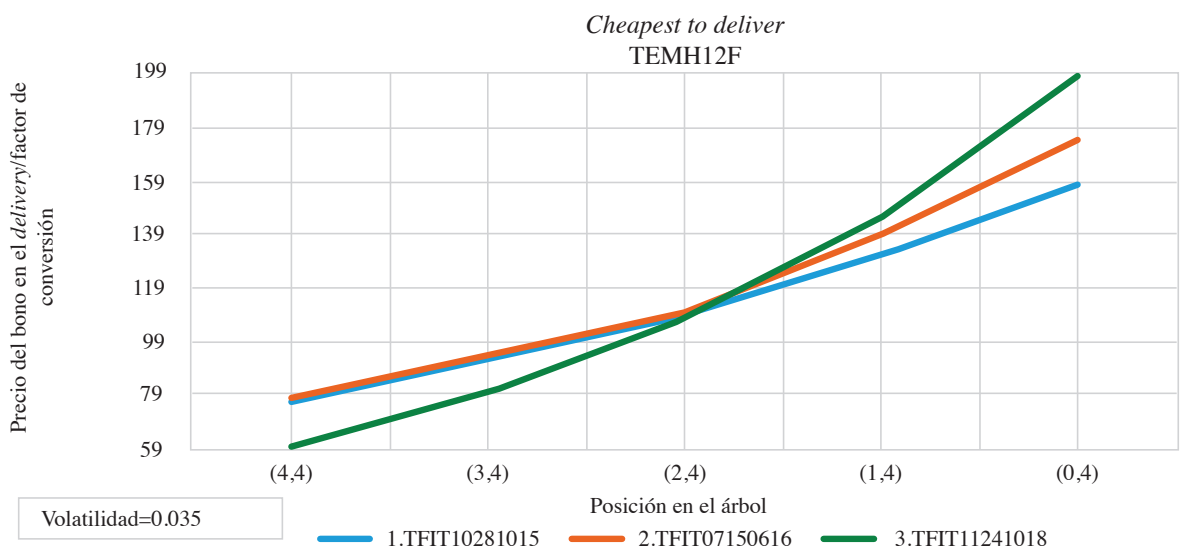



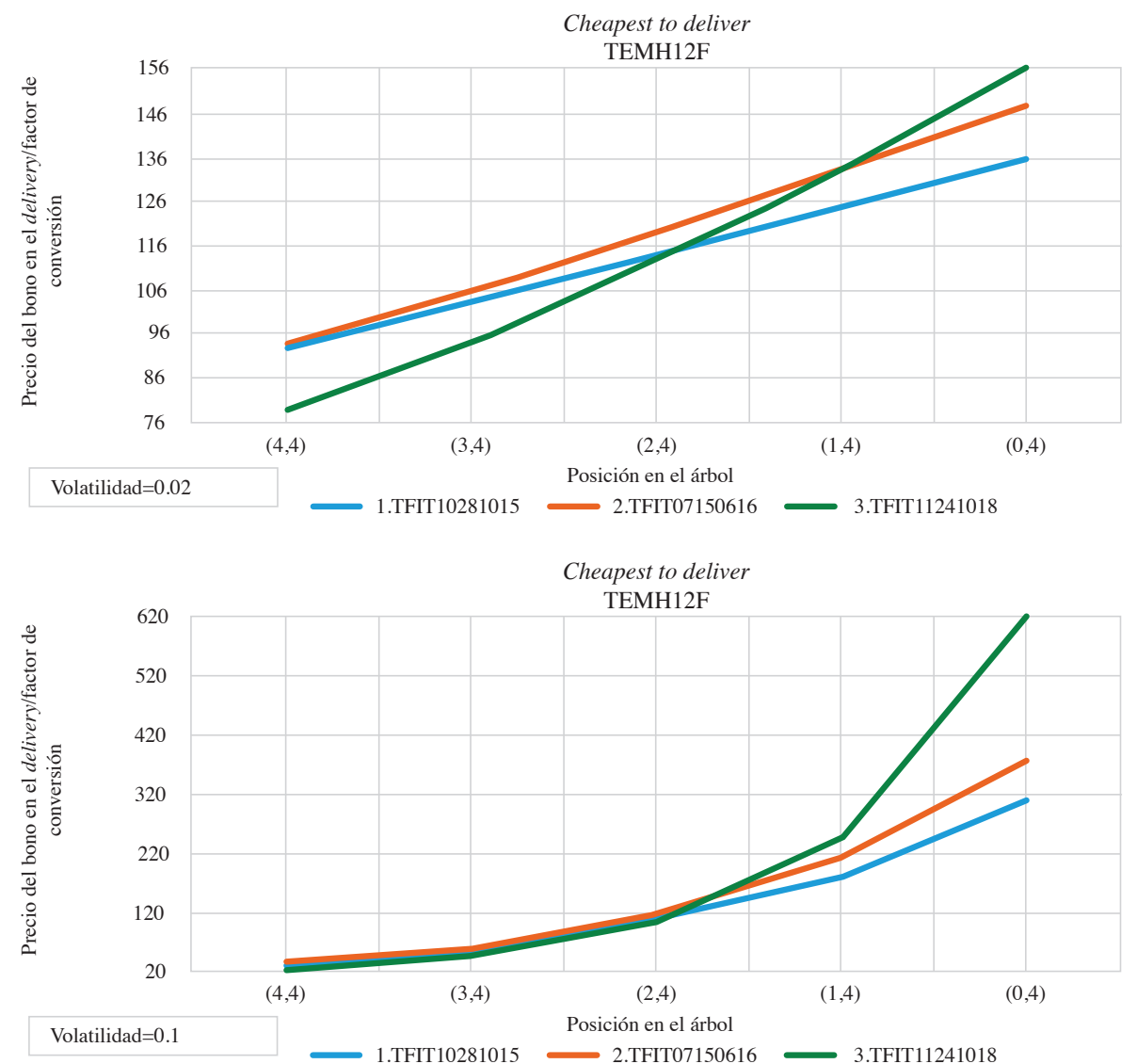

\subsubsection{Resultados de la metodología para otros contratos}

La metodología se aplicó de la misma forma explicada en las secciones precedentes y siguiendo este orden:

a) Cálculo de la curva cero cupón y tasas intermedias.

b) Construcción del árbol de tasas Ho-Lee.

c) Cálculo de los factores de descuento y sus tasas equivalentes.

d) Cálculo del precio del bono en la fecha del delivery.

e) Cálculo del CTD y construcción del árbol de precios del futuro y el árbol de probabilidades.

f) Análisis de sensibilidad del CTD vs. cambios en la CCC.

g) Análisis de sensibilidad del CTD vs. cambios en la volatilidad. 


\subsubsection{Contrato corto plazo: TESH13F}

Tabla 14: Características del futuro por analizar

\begin{tabular}{|c|c|}
\hline \multicolumn{2}{|c|}{ Datos } \\
\hline Contrato & TESH13F \\
\hline Primera Negociación & $20 / 11 / 2012$ \\
\hline Fecha Actual & $20 / 11 / 2012$ \\
\hline Fecha delivery & $27 / 02 / 2013$ \\
\hline Factores de conversión de entregables \\
\hline TFIT06140514 & 1,03 \\
\hline TFIT10281015 & 1,04 \\
\hline
\end{tabular}

Fuente: elaboración propia.

Los resultados de este ejercicio muestran que el CTD cambia en los nodos del árbol en la fecha de delivery, siendo el TFIT06140514 el CTD en los nodos con las tasas más bajas $([1,4]$ y $[0,4])$ y el TFIT10281015 resultó el CTD en los nodos con las tasas más altas $([4,4],[3,4]$ y $[2,4])$, estos hallazgos son coherentes con lo observado en la figura 7 .

Para determinar los efectos en el CTD de los movimientos de las tasas se analizaron cinco escenarios en los que se modificó el valor de la CCC: 1) CCC+100 p.b.; 2) CCC-100 p.b.; 3) CCC+200 p.b.; 4) CCC-200 p.b., y 5) CCC - 300 p.b. (figura 9). Si bien se evidencian cambios en el valor del futuro, el CTD en cada nodo es el mismo que resulta con los datos iniciales. Adicionalmente, es importante resaltar que se mantienen las relaciones expuestas en la figura 7.

En el análisis de sensibilidad del CTD respecto a cambios en la volatilidad se manejaron seis escenarios en los que se modificó esta variable: 1) 0,01 ; 2) 0,015 ; 3) 0,02 ; 4) 0,025 ; 5) 0,04 , y 6) 0,1 . A diferencia del contrato de mediano plazo, a medida que se incrementa el valor de la volatilidad, el TFIT10281015 deja de ser el CTD en todos los nodos del árbol, y el TFIT06140514 se convierte en el CTD en los nodos de las tasas más bajas $([0,4]$ y $[1,4])$; por encima de una volatilidad del 0,1 no se evidencian más cambios en el CTD para cada nodo del árbol (figura 10).

Para consultar los resultados en detalle ver Anexo 2. 
4.4.2.2. Contrato largo plazo: TELJ10F T4

Tabla 15: Características del futuro por analizar

\begin{tabular}{|c|c|}
\hline \multicolumn{2}{|c|}{ Datos } \\
\hline Contrato & TELJ10F \\
\hline Primera Negociación & $11 / 02 / 2010$ \\
\hline Fecha Actual & $11 / 02 / 2010$ \\
\hline Fecha delivery & $05 / 04 / 2010$ \\
\hline \multicolumn{2}{|c|}{ Factores de conversión de entregables } \\
\hline TFIT15240720 & 1,204057 \\
\hline TFIT16240724 & 1,165523 \\
\hline
\end{tabular}

Fuente: elaboración propia.

Los resultados de este ejercicio muestran que el CTD no cambia en los nodos del árbol en la fecha de delivery, y siempre corresponde al TFIT16240724. Esto obedece a que las tasas del árbol Ho-Lee no abarcan el rango en el cual cambia el CTD, de acuerdo con lo que se observa en la figura 11.

Para determinar los efectos en el CTD de los movimientos de las tasas se analizaron cinco escenarios en los que se modificó el valor de la CCC: 1) CCC+100 p.b.; 2) CCC-100 p.b.; 3) CCC-200 p.b.; 4) CCC-300 p.b., y 5) CCC - 325 p.b. (figura 13). Se identifica que a medida que disminuyen las tasas de la curva cero cupón, el TFIT16240724 deja de ser el CTD en todos los nodos del árbol y el TFIT15240720 se convierte en el CTD en los nodos que corresponden a las tasas más bajas, incluso en el último escenario esta referencia es el CTD en cuatro no$\operatorname{dos}([0,4],[1,4],[2,4],[3,4])$.

En el análisis de sensibilidad del CTD respecto a cambios en la volatilidad, se manejaron seis escenarios en los que se modificó esta variable: 1) 0,01 ; 2) 0,015 ; 3) 0,02 ; 4) 0,035 ; 5) 0,045 , y 6) 0,1 . A partir de una volatilidad de 0,015 el CTD en los nodos de tasas más bajas $([1,4],[0,4])$ es el TFIT15240720, y en los restantes es el TFIT16240724 (figura 14).

Para consultar los resultados en detalle ver Anexo 3.

\section{Conclusiones}

De acuerdo con los casos analizados en las secciones anteriores se puede concluir que: 
- Los contratos de corto y mediano plazo presentan cambios del CTD en los nodos del árbol en la fecha del delivery, con la particularidad de que en el contrato de mediano plazo uno de los tres entregables nunca se convierte en el CTD.

- Para el contrato de largo plazo la implementación de la metodología muestra el mismo entregable como CTD en todos los nodos en la fecha del delivery. Este resultado va en contravía de la intuición que apunta a que el CTD para los contratos de mayor plazo podría ser el más difícil de predecir, debido a que a mayor plazo el precio de los bonos es más sensible a los cambios en el nivel de tasas.

- El resultado obtenido para el contrato de largo plazo podría obedecer a que la volatilidad de la tasa de interés de corto plazo se calculó con información histórica de las tasas de retorno diarias de los entregables, en lugar de usar la volatilidad implícita en contratos de opciones sobre títulos de deuda pública, como es lo recomendado en estos casos. Se siguió este procedimiento debido a la no disponibilidad de opciones sobre títulos de deuda pública en el mercado colombiano.

- Otro factor que pudo afectar los resultados corresponde a la utilización de tasas del mercado monetario en la construcción de la curva cero cupón, debido a la no disponibilidad de títulos de deuda pública con vencimientos en el corto plazo.

- El análisis de sensibilidad del CTD respecto al nivel de la curva de tasas muestra que:

- En el contrato de corto plazo no se evidencian cambios en el CTD, los resultados son iguales a los obtenidos con los datos iniciales.

- En el contrato de mediano plazo no se evidencian cambios en el CTD, los resultados son iguales a los obtenidos con los datos iniciales.

- En el contrato de largo plazo el TFIT16240724 deja de ser el CTD en todos los nodos del árbol cuando aumenta la tasa.

- El análisis de sensibilidad del CTD respecto al valor de la volatilidad muestra que:

- En el contrato de corto plazo, el TFIT10281015 deja de ser el CTD en todos los nodos del árbol, y el TFIT06140514 se convierte en el CTD en los nodos de las tasas más bajas.

- En el contrato de mediano plazo no se evidencian cambios en el CTD, los resultados son iguales a los obtenidos con los datos iniciales. 
- En el contrato de largo plazo, el TFIT16240724 deja de ser el CTD en todos los nodos del árbol cuando aumenta la volatilidad.

- Una extensión del presente trabajo consiste en realizar el análisis de sensibilidad del CTD frente a cambios en el nivel de tasas, contemplando choques no paralelos que se aproximen más a los cambios de pendiente y curvatura que se observan en el mercado.

\section{Referencias}

Bolsa de Valores de Colombia (2014). Circular única del mercado de derivados de la Bolsa de Valores de Colombia S.A. Recuperado de www.bvc.com.co

Cámara de Riesgo Central de Contraparte de Colombia S.A. (2014). Circular única de la Cámara de Riesgo Central de Contraparte de Colombia. Recuperado de www. camaraderiesgo.com

Cox, J. C., Ingersoll Jr, J. E. y Ross, S. A. (1980). An analysis of variable rate loan contracts. The Journal of Finance, 35(2), 389-403.

Cox, J. C., Ingersoll Jr, J. E. y Ross, S. A. (1985). An intertemporal general equilibrium model of asset prices. Econometrica: Journal of the Econometric Society, 363-384.

Chan, A. K. C., Karolyi, G. A., Longstaff, F. A., Sanders, A. B. y Longstaff, F. A. (2014). An empirical comparison of alternative models of the short-term interest rate. The Journal of Finance, 47(3).

Choudhry, M. (2010). The Repo Handbook (2 ed.). Butterworth-Heinemann.

Dothan, L. U. (1978). On the term structure of interest rates. Journal of Financial Economics, 6(1), 59-69.

Flesaker, B. (1993). Testing the Heath-Jarrow-Morton / Ho-Lee Model of interest rate contingent claims pricing author. Journal of Financial and Quantitative Analysis, 28(4), 483-495.

Grant, D. y Vora, G. (2003). Analytical implementation of the Ho and Lee model for the short interest rate. Global Finance Journal, 14(1), 19-47.

Grieves, R. y Marcus, A. (2005). Delivery options and treasury bond futures hedge ratios. Journal of Derivatives, 13, 70-76. 
Grieves, R., Marcus, A. J. y Woodhams, A. (2010). Review of financial economics delivery options and convexity in treasury bond and note futures. Review of Financial Economics, 19(1), 1-7. doi:10.1016/j.rfe.2009.06.003

Hagan, P. y West, G. (2008). Methods for constructing a yield curve. Wilmott Magazine, 70-81.

Henrard, M. P. A. (2006). Bond Futures and Their Options: More than the Cheapestto-Deliver. Margining and Quality Option. Recuperado de https://ssrn.com/abstract=881741 or http://dx.doi.org/10.2139/ssrn.881741

Ho, T. S. Y. y Lee, S.-B. (1986). Term structure movements and pricing interest rate contingent claims. Journal of Finance, 41(5), 1011-1029.

Ho, T. S. Y. y Lee, S.-B. (2004). The Oxford Guide to Financial Modeling. Applications for Capital Markets, Corporate Finance, Risk Management and Financial Institutions. Oxford: Oxford University Press.

Hérault, A. R., Julio, J. M. y Mera, S. J. (2002). La curva spot (cero cupón), Estimación con splines cúbicos suavizados, usos y ejemplos (No. 002961). Bogotá: Banco de la República.

Leippold, M. y Wiener, Z. (1992). Algorithms behind Term Structure Models of Interest Rates I . Valuation and Hedging of Interest Rates Derivatives with the Ho-Lee Model, 1-20 (Working Paper).

López García, M. y Valderrama Guzmán, Á. (2008). Futuros de TES y curva de rendimientos: estrategias y requerimientos. Publicación Técnica de la Bolsa de Valores de Colombia, 1-27. Recuperado de www.bvc.com.co

van Straaten, M. (2009). Determining the Cheapest-to-Deliver Bonds for Bond Futures. Utrecht: Utrecht University.

Zazaravaka Rahantamialisoa, T. F. (2010). Ho and Lee Model of the Term Structure of Interest Rates. African Institute for Mathematical Sciences. Recuperado de http:// users.aims.ac.za/ tahiri/PGD-Essay-Template-2009_10.pdf

Zbtprice (n. d.). MathWorks. Recuperado de https://es.mathworks.com/help/finance/ zbtprice.html 


\section{Anexos}

\section{Anexo 1}

\section{Función zbtprice de MatLab}

La función utiliza la metodología del bootstrapping para calcular la curva cero cupón con base en un portafolio de bonos con cupón y sus respectivos precios. La curva cero cupón contiene las tasas hasta el vencimiento para un portafolio teórico de bonos cero cupón, que son calculados a partir de la información del portafolio de bonos de entrada. La metodología de bootstrapping que utiliza la función no requiere que exista alineación entre las fechas de pago de cupones de los bonos del portafolio de entrada.

A continuación se presentan los parámetros de entrada de la función:

1. Bonos: matriz que contiene la información del portafolio de bonos de entrada, que incluye: fecha al vencimiento (obligatorio), tasa cupón (obligatorio), valor facial (opcional), número de pagos de cupón al año (opcional), sistema de conteo de días (opcional), regla de fin de mes (opcional).

2. Precios: vector columna que contiene el precio limpio para cada uno de los bonos de entrada.

3. Settle: fecha de cálculo de la curva o fecha actual.

4. OutputCompounding: valor escalar que representa el periodo en el cual se componen las tasas cero cupón.

A continuación se presenta la información de salida de la función:

1. Tasas cero cupón: vector que contiene las tasas cero cupón correspondientes a cada fecha de vencimiento de los bonos de entrada suministrados.

2. Fechas: vector que muestra las fechas a las que corresponden las tasas de salida, las fechas son las de vencimiento para cada uno de los bonos de entrada suministrados.

Ejemplo de uso de la función: 


\section{INFORMACIÓN DE ENTRADA}

Bonds $=$ [datenum('6/1/1998') 0,0475 10020 0;

datenum('7/1/2000') 0,06 100200 ;

datenum('7/1/2000') 0,09375 100610 ;

datenum('6/30/2001') 0,05125100131;

datenum('4/15/2002') 0,07125100410;

datenum(' $1 / 15 / 2000$ ') 0,065 100200 ;

datenum('9/1/1999') 0,08 100330 ;

datenum('4/30/2001') 0,05875100200;

datenum('11/15/1999') 0,07125100200;

datenum('6/30/2000') 0,07 100231 ;

datenum('7/1/2001') 0,0525100230;

datenum('4/30/2002') 0,07 10020 0];

Prices $=[99.375$

99.875;

105.75;

96.875

103.625;

101.125;

103.125;

99.375;

101.0

101.25;

96.375

102.75];

Settle = datenum('12/18/1997');

OutputCompounding $=2$;

\section{Aplicación de la función}

[ZeroRates, CurveDates] = zbtprice $($ Bonds, Prices, Settle,...OutputCompounding $)$ 


\section{Información de salida de la función}

ZeroRates $=$

0,0616

0,0609

0,0658

0,0590

0,0647

0,0655

0,0606

0,0601

0,0642

0,0621

0,0627

CurveDates $=$

729907

730364

730439

730500

730667

730668

730971

731032

731033

731321

731336

Información tomada de la página web de MathWorks el día 14 de febrero del 2017. Enlace de consulta: https://es.mathworks.com/help/finance/zbtprice.html

\section{Anexo 2}

Paso a paso de la implementación para el contrato de corto plazo: TESH13F

Antes de implementar la metodología, y con el objeto de identificar si el CTD cambia para diferentes tasas, se calculó el precio del futuro en la fecha actual 
para diferentes niveles de tasa (yield). En la figura 7 se observa que el entregable TFIT06140514 es el CTD cuando la tasa disminuye, mientras que el TFIT10281015 se convierte en el CTD cuando la tasa aumenta.

Figura 7: Precio del futuro vs. movimientos en las tasas para el contrato TESH13F

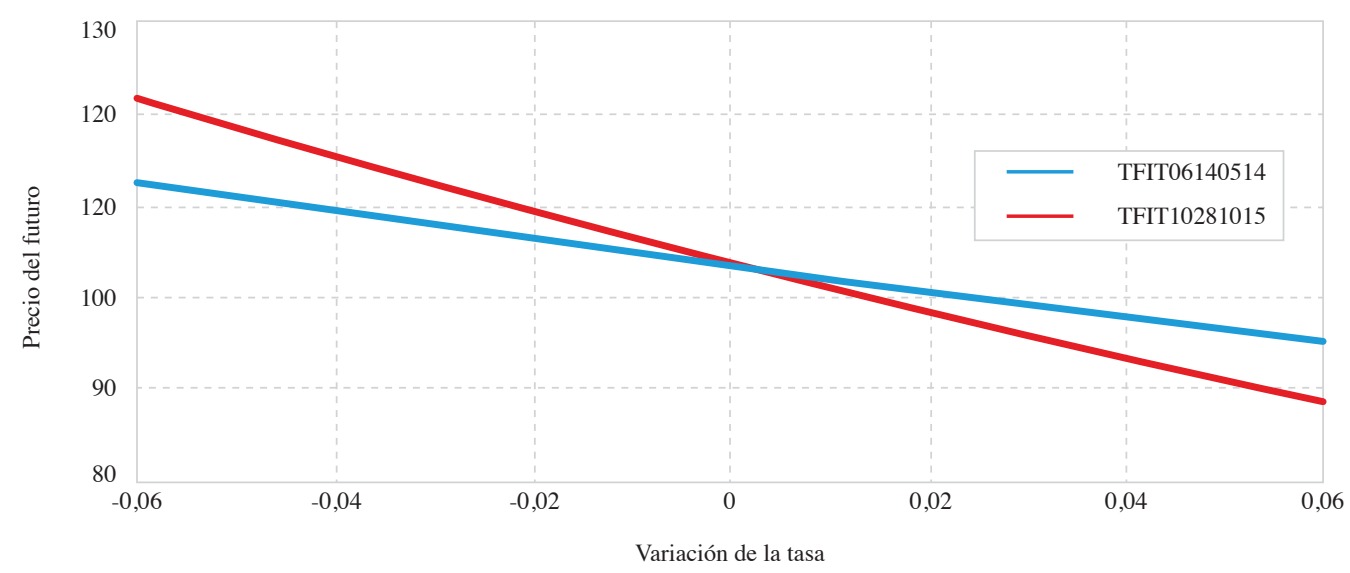

Tabla 16: Información para construir la curva cero cupón

\begin{tabular}{|c|c|}
\hline \multicolumn{2}{|c|}{ Datos monetarios } \\
\hline IBR overnight & 0,0475 \\
\hline IBR 30 días & 0,0474 \\
\hline IBR 90 días & 0,0471 \\
\hline
\end{tabular}

\begin{tabular}{|c|c|c|c|c|}
\hline Títulos del mercado & Precios de cierrre & Cupón & Fecha de vencimiento & Días al vencimiento \\
\hline TFIT04170413 & 100,304 & 6 & $17 / 04 / 13$ & 148 \\
\hline TFIT06140514 & 105,640 & 9,25 & $14 / 05 / 14$ & 540 \\
\hline TFIT10281015 & 106,920 & 8 & $28 / 10 / 15$ & 1072 \\
\hline TFIT07150616 & 105,559 & 7,25 & $15 / 06 / 16$ & 1303 \\
\hline TFIT11241018 & 127,339 & 11,25 & $24 / 10 / 18$ & 2164 \\
\hline TFIT15240720 & 130,869 & 11 & $24 / 07 / 20$ & 2803 \\
\hline TFIT10040522 & 106,562 & 7 & $4 / 05 / 22$ & 3452 \\
\hline TFIT16240724 & 131,248 & 10 & $24 / 07 / 24$ & 4264 \\
\hline TFIT15260826 & 110,394 & 7,5 & $26 / 08 / 26$ & 5027 \\
\hline
\end{tabular}

Fuente: elaboración propia. 
La curva cero cupón calculada para este caso tiene la siguiente forma (tabla 17):

Tabla 17: Curva cero cupón - Contrato TESH13F

\begin{tabular}{|c|c|c|}
\hline Días al vencimiento & Años & Tasa \\
\hline 1 & 0,002739726 & 0,047940113 \\
\hline 30 & 0,082191781 & 0,047504883 \\
\hline 90 & 0,246575342 & 0,047463291 \\
\hline 148 & 0,405479452 & 0,049897913 \\
\hline 540 & 1,479452055 & 0,050095828 \\
\hline 1072 & 2,936986301 & 0,052601444 \\
\hline 1303 & 3,569863014 & 0,053518482 \\
\hline 2164 & 5,928767123 & 0,055872453 \\
\hline 2803 & 7,679452055 & 0,058124458 \\
\hline 3452 & 9,457534247 & 0,059892017 \\
\hline 4264 & 11,68219178 & 0,061480715 \\
\hline 5027 & 13,77260274 & 0,063519803 \\
\hline
\end{tabular}

Fuente: elaboración propia.

Figura 8: Curva cero cupón - Contrato TESH13F

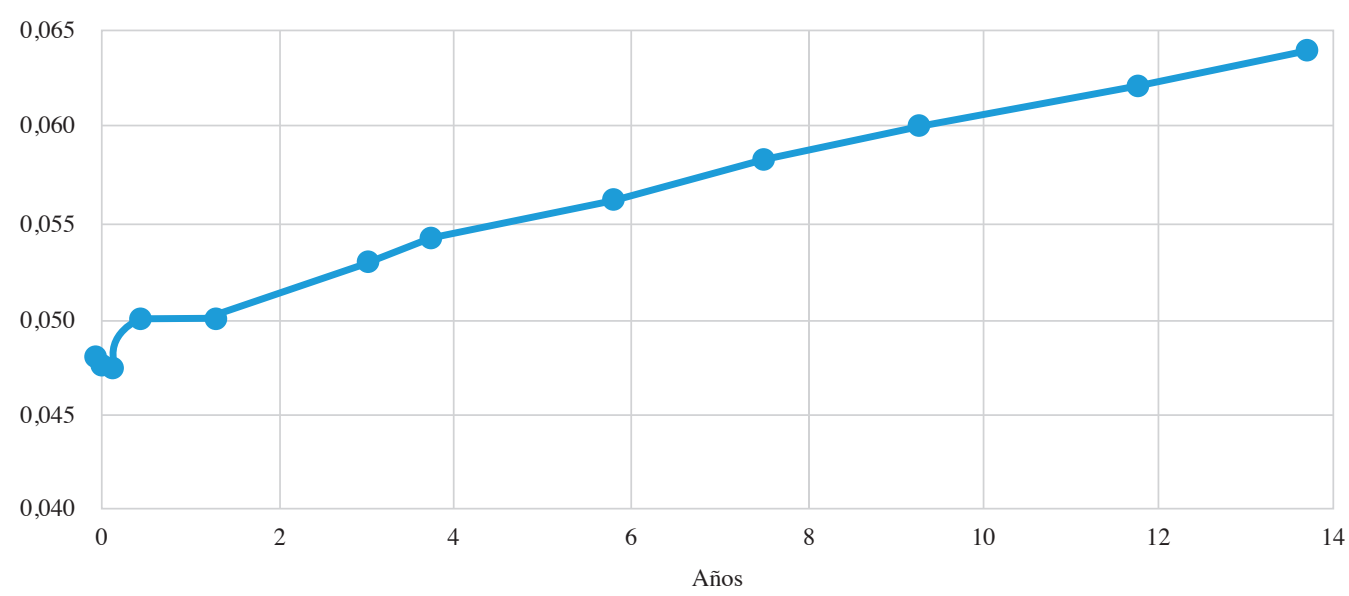


En la construcción de la curva cero cupón se incluyó información del mercado oIS para los plazos de 1, 30 y 90 días, con el fin de tener referencias de corto plazo que permitieran realizar interpolación para hallar las tasas correspondientes a los pasos del árbol Ho-Lee.

Tabla 18: Información para la construcción del árbol Ho-Lee

\begin{tabular}{|c|c|}
\hline Número de días desde la fecha actual hasta la fecha del delivery & 99 \\
\hline Número de pasos del árbol & 4 \\
\hline Número de días de cada periodo & 24,75 \\
\hline Volatilidad & 0,128201 \\
\hline Delta & 0,987892698 \\
\hline
\end{tabular}

Fuente: elaboración propia.

Con base en la CCC calculada, y utilizando el método de interpolación log linear on the discount factors (ecuación 3), se calculan las tasas cero cupón para los respectivos nodos del árbol (tabla 19):

Tabla 19: Cálculos intermedios en la construcción del árbol de tasas de Ho-Lee

\begin{tabular}{|c|c|c|}
\hline Pasos & Días & Tasas cero cupón \\
\hline 1 & 24,750 & 0,04751 \\
\hline 2 & 49,500 & 0,04748 \\
\hline 3 & 74,250 & 0,04747 \\
\hline 4 & 99,000 & 0,04803 \\
\hline 5 & 123,75 & 0,04916 \\
\hline
\end{tabular}

\begin{tabular}{|c|c|}
\hline Pasos & Precio del bono P(n) \\
\hline 1 & 0,99678375 \\
\hline 2 & 0,99358160 \\
\hline 3 & 0,99039037 \\
\hline 4 & 0,98705769 \\
\hline 5 & 0,983447167 \\
\hline
\end{tabular}

Fuente: elaboración propia. 
Tabla 20: Árbol de tasas por medio de metodología Ho-Lee

\begin{tabular}{|c|c|c|c|c|c|c|c|c|}
\hline Hoy & & 1 & & 2 & & 3 & & 4 \\
\hline \multirow[t]{5}{*}{$4,75 \%$} & 1 & $13,75 \%$ & 2 & $22,82 \%$ & 3 & $32,16 \%$ & 4 & $41,73 \%$ \\
\hline & 0 & $-4,21 \%$ & 1 & $4,85 \%$ & 2 & $14,20 \%$ & 3 & $23,77 \%$ \\
\hline & & & 0 & $-13,11 \%$ & 1 & $-3,77 \%$ & 2 & $5,81 \%$ \\
\hline & & & & & 0 & $-21,73 \%$ & 1 & $-12,16 \%$ \\
\hline & & & & & & & 0 & $-30,12 \%$ \\
\hline
\end{tabular}

Fuente: elaboración propia.

A continuación se presentan los resultados de los factores de descuento, las tasas equivalentes y precios del futuro utilizando la misma volatilidad, calculada como la mayor desviación estándar de las tasas de retorno históricas de los dos entregables.

Tabla 21: Factores de descuento para cada flujo y posición en el árbol - Contrato TESH13F

\begin{tabular}{|c|c|c|c|c|c|c|}
\hline \multicolumn{7}{|c|}{ Factores de descuento } \\
\hline & \multirow{7}{|c|}{ Posición en el árbol } \\
\cline { 2 - 8 } & Flujos & $(4,4)$ & $(3,4)$ & $(2,4)$ & $(1,4)$ & $(0,4)$ \\
\hline TFIT06140514 & 1 & 0,91584295 & 0,9507487 & 0,98698481 & 1,024602 & 1,0636529 \\
\hline & 2 & 0,60016862 & 0,74565077 & 0,92639811 & 1,15095898 & 1,42995388 \\
\cline { 2 - 8 } & & \multicolumn{5}{|c|}{ Posición en el árbol } \\
\cline { 2 - 8 } & Flujos & $(4,4)$ & $(3,4)$ & $(2,4)$ & $(1,4)$ & $(0,4)$ \\
\hline TFIT 10281015 & 1 & 0,75523463 & 0,85118172 & 0,95931818 & 1,08119259 & 1,21855027 \\
\hline & 2 & 0,49278784 & 0,66468798 & 0,89655239 & 1,2092985 & 1,63114046 \\
\cline { 2 - 7 } & 3 & 0,31924946 & 0,51535379 & 0,83191848 & 1,34293834 & 2,1678607 \\
\hline
\end{tabular}

Fuente: elaboración propia.

A partir de los factores de descuentos de la tabla 21 es posible calcular las tasas equivalentes y con ellas construir una curva de tasas para cada nodo final del árbol. Estas curvas se extienden desde la fecha del delivery hasta la fecha del último flujo de los entregables del contrato de futuro. Dichas tasas se calcularon usando la ecuación 29. 
Tabla 22: Árbol de tasas Ho-Lee - Contrato TESH13F

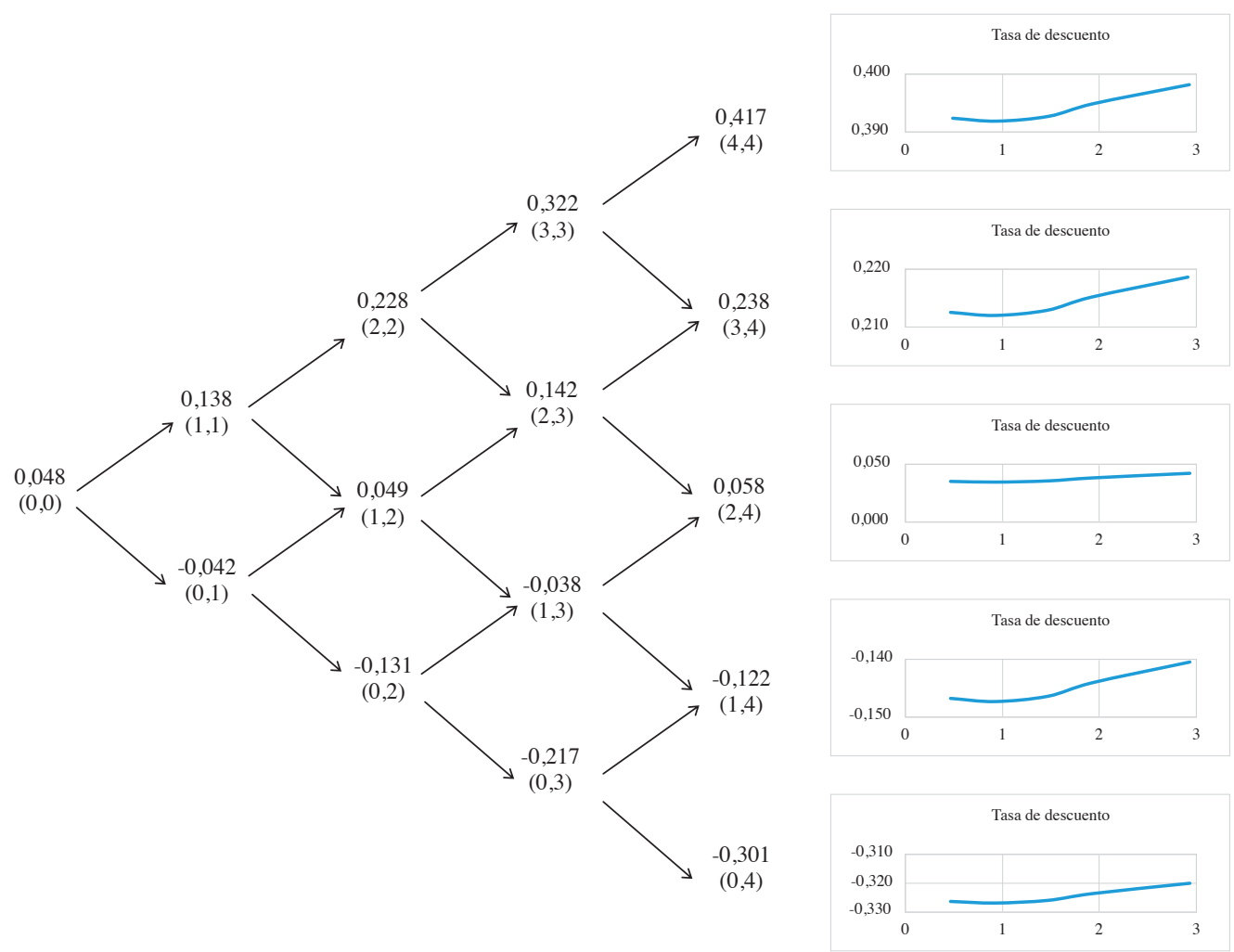

Fuente: elaboración propia.

Tabla 23: Precio de los bonos entregables en la fecha del delivery - Contrato TESH13F

\begin{tabular}{|c|c|c|c|c|c|c|}
\hline \multicolumn{7}{|c|}{ Precio de los bonos en la fecha del delivery } \\
\hline Fecha actual & \multicolumn{2}{|c|}{$20 / 11 / 12$} & & & & \\
\hline Última fecha que pagó cupón & \multicolumn{2}{|c|}{$14 / 05 / 12$} & & & & \\
\hline Cupón & \multicolumn{2}{|c|}{9,25} & & & & \\
\hline Número de días & \multicolumn{2}{|c|}{190} & & & & \\
\hline \multirow[t]{3}{*}{ Intereses devengados } & \multicolumn{2}{|c|}{4,815068493} & & & & \\
\hline & \multicolumn{6}{|c|}{ Posición en el árbol } \\
\hline & Flujos & $(4,4)$ & $(3,4)$ & $(2,4)$ & $(1,4)$ & $(0,4)$ \\
\hline \multirow[t]{2}{*}{ TFIT06140514 } & 1 & 8,47154732 & 8,79442544 & 9,1296095 & 9,47756851 & 9,83878936 \\
\hline & 2 & 65,5684221 & 81,462347 & 101,208993 & 125,742268 & 156,222462 \\
\hline
\end{tabular}




\begin{tabular}{|c|c|c|c|c|c|c|}
\hline Precio Sucio & & 74,03997 & 90,25677 & 110,33860 & 135,21984 & 166,06125 \\
\hline Precio Limpio & & 69,22490 & 85,44170 & 105,52353 & 130,40477 & 161,24618 \\
\hline Fecha actual & \multicolumn{2}{|c|}{$20 / 11 / 12$} & & & & \\
\hline Última fecha que pagó cupón & \multicolumn{2}{|c|}{$28 / 10 / 12$} & & & & \\
\hline Cupón & \multicolumn{2}{|r|}{8} & & & & \\
\hline Número de días & \multicolumn{2}{|r|}{23} & & & & \\
\hline \multirow[t]{3}{*}{ Intereses devengados } & \multicolumn{2}{|c|}{0,504109589} & & & & \\
\hline & \multicolumn{6}{|c|}{ Posición en el árbol } \\
\hline & Flujos & $(4,4)$ & $(3,4)$ & $(2,4)$ & $(1,4)$ & $(0,4)$ \\
\hline \multirow[t]{3}{*}{ TFIT10281015 } & 1 & 6,04187707 & 6,80945372 & 7,67454543 & 8,64954076 & 9,74840217 \\
\hline & 2 & 3,94230273 & 5,31750386 & 7,1724191 & 9,67438802 & 13,0491236 \\
\hline & 3 & 34,4789418 & 55,6582091 & 89,847196 & 145,037341 & 234,128955 \\
\hline Precio Sucio & & 44,46312 & 67,78517 & 104,69416 & 163,36127 & 256,92648 \\
\hline Precio Limpio & & 43,95901 & 62,97010 & 99,87909 & 158,54620 & 252,11141 \\
\hline
\end{tabular}

Fuente: elaboración propia.

Tabla 24: Precio del CTD en la fecha del delivery - Contrato TESH13F

\begin{tabular}{|c|c|c|c|c|c|c|c|c|}
\hline \multicolumn{7}{|c|}{ Precio de los bonos en la fecha del delibery } \\
\cline { 4 - 9 } & \multicolumn{7}{|c|}{ Posición en el árbol } \\
\cline { 4 - 9 } & $(4,4)$ & $(3,4)$ & $(2,4)$ & $(1,4)$ & $(0,4)$ \\
\hline 1. TFIT06140514 & Precios & & 69,225 & 85,442 & 105,524 & 130,405 & 161,246 \\
\hline & FC & 1,03 & \multicolumn{7}{|c|}{} \\
\cline { 2 - 9 } & Precio del Futuro & & 67,209 & 82,953 & 102,450 & 126,607 & 156,550 \\
\hline 2. TFIT10281015 & Precios & & 43,959 & 62,970 & 99,879 & 158,546 & 252,111 \\
\hline & FC & 1,04 & & 42,268 & 60,548 & 96,038 & 152,448 & 242,415 \\
\cline { 2 - 9 } & Precio del Futuro & & 42,268 & 60,548 & 96,038 & 126,607 & 156,550 \\
\hline
\end{tabular}

Fuente: elaboración propia. 
Tabla 25: Árbol de precios del futuro - Contrato TESH13F

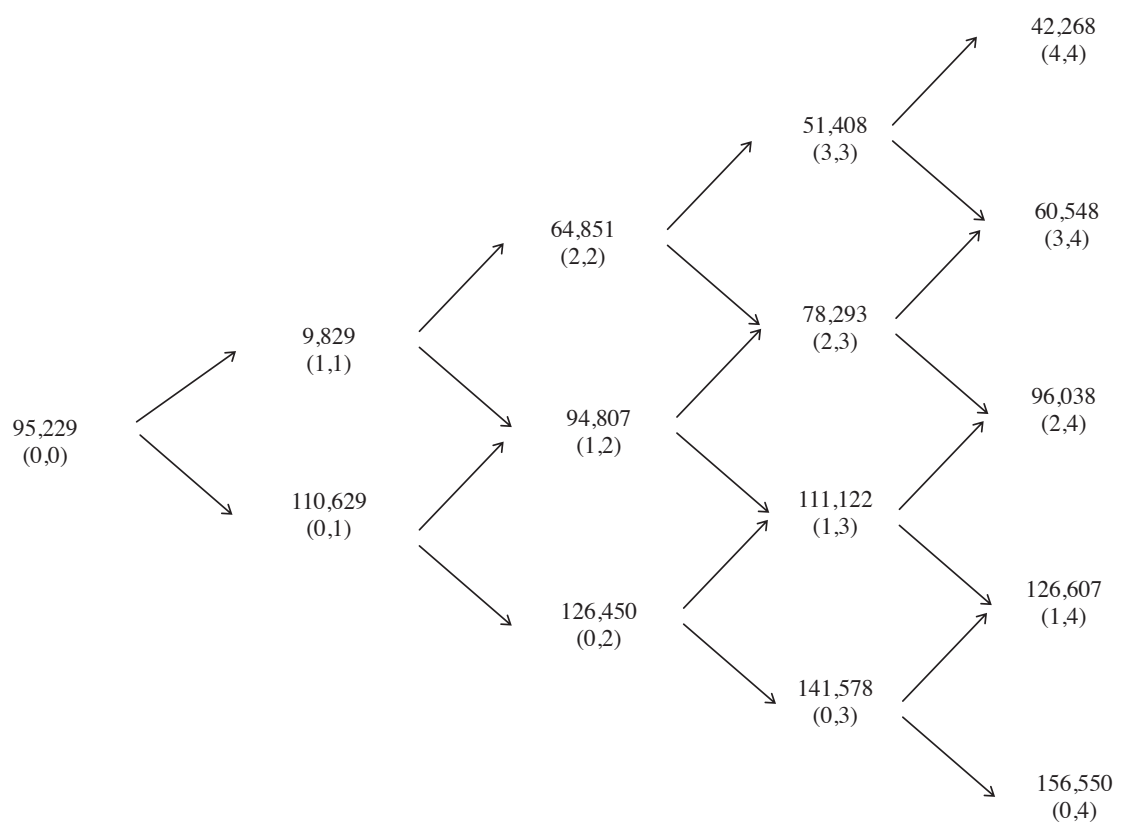

Fuente: elaboración propia.

Tabla 26: Árbol de probabilidades del CTD - Contrato TESH13F

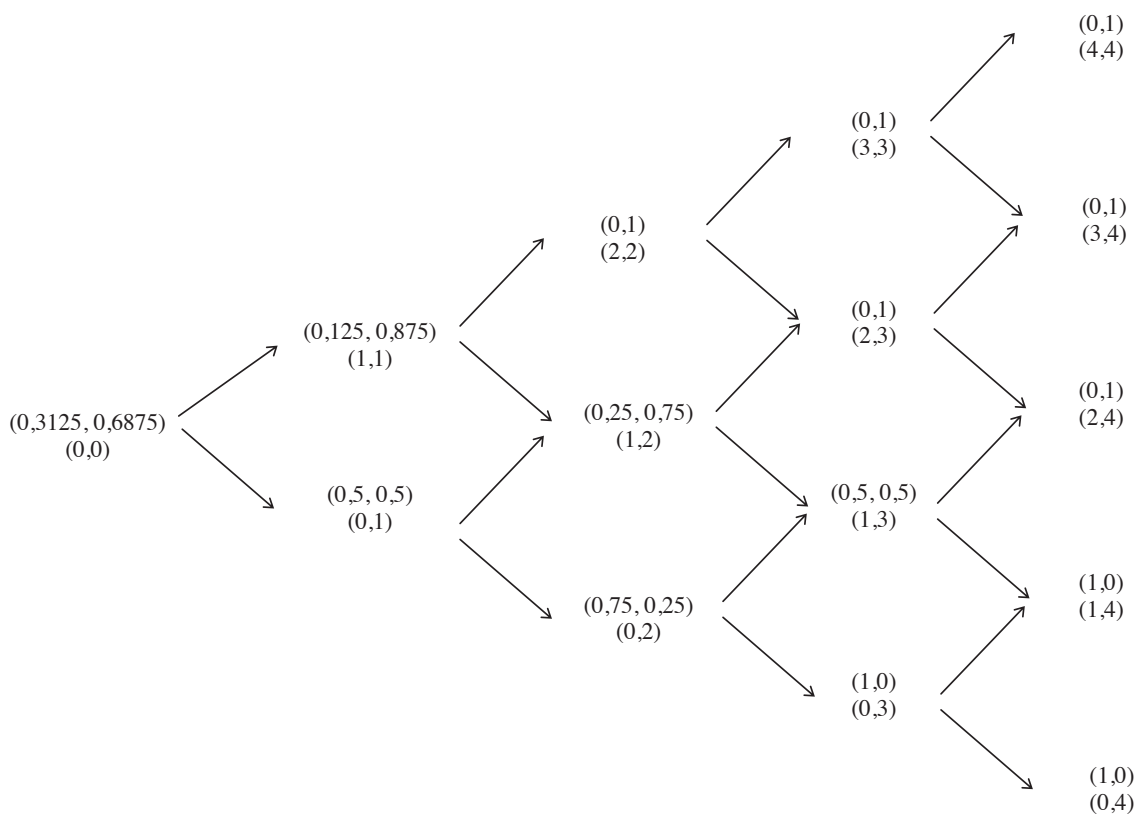

Fuente: elaboración propia. 
Figura 9: Análisis de sensibilidad del CTD ante cambios en la curva cero cupón - Contrato TESH13F
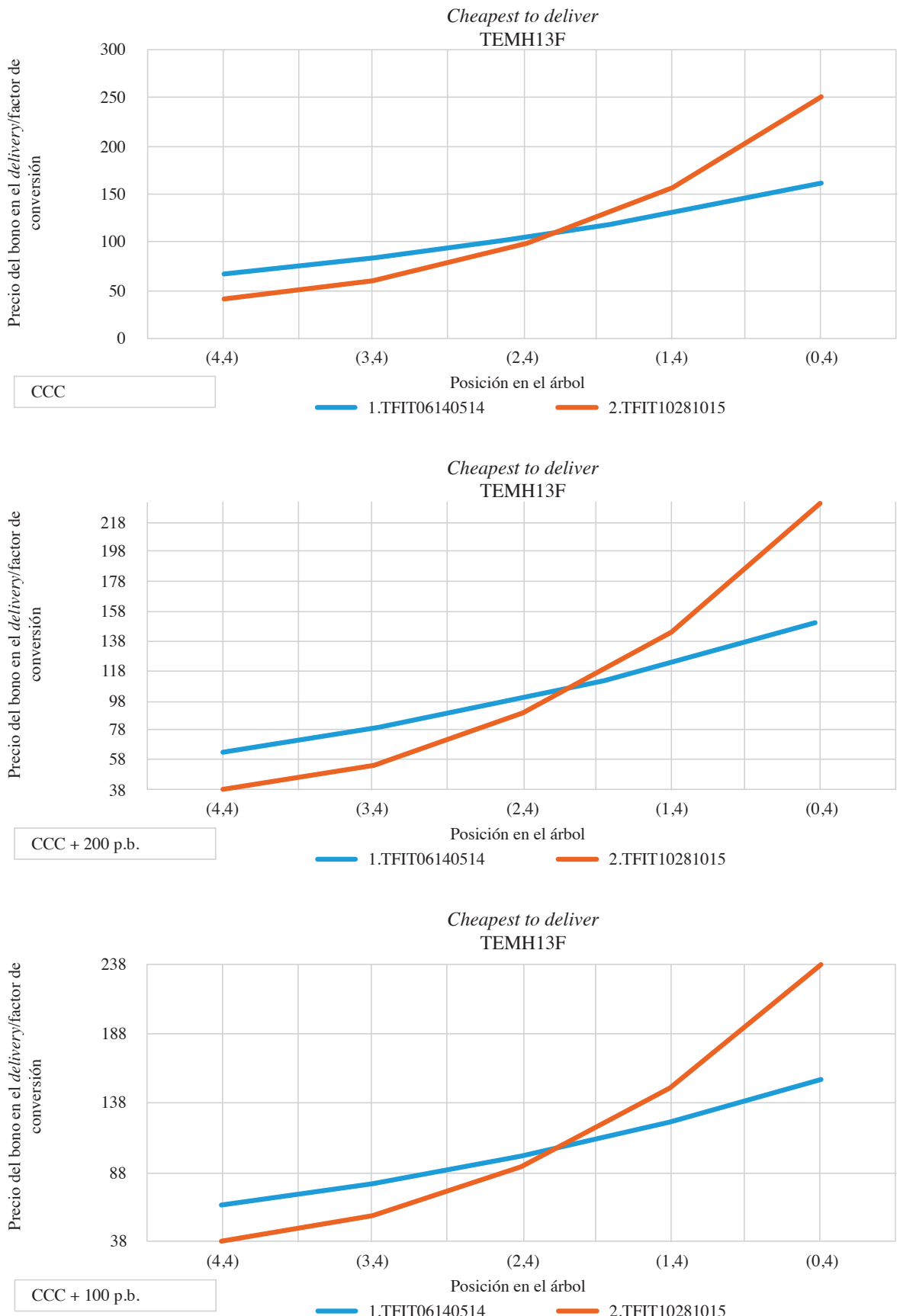

ODEON, ISSN: 1794-1113, E-ISSN: 2346-2140, N 15, julio-diciembre de 2018, pp. 73-137 

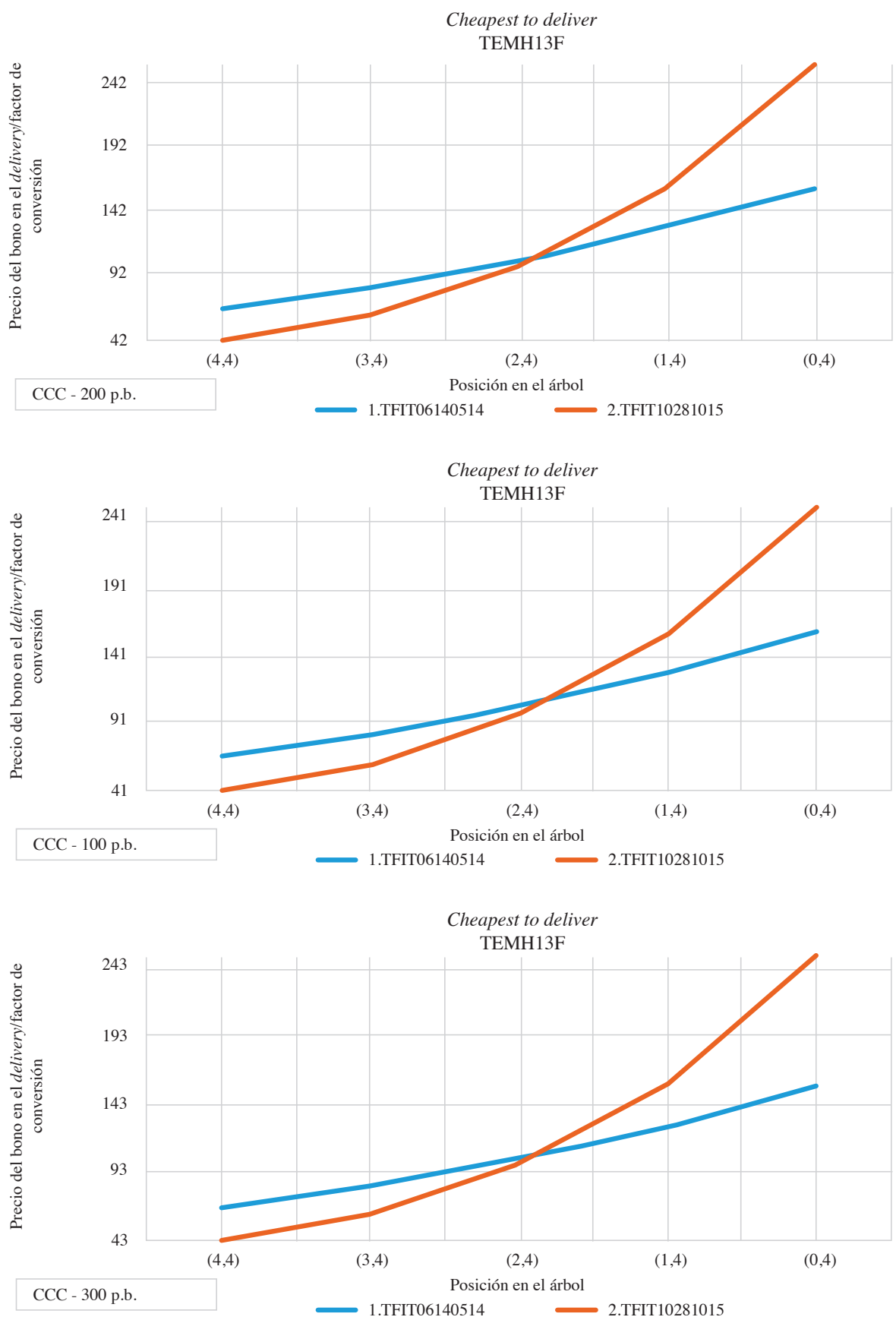
Figura 10: Análisis de sensibilidad del CTD ante cambios en la volatilidad - Contrato TESH13F
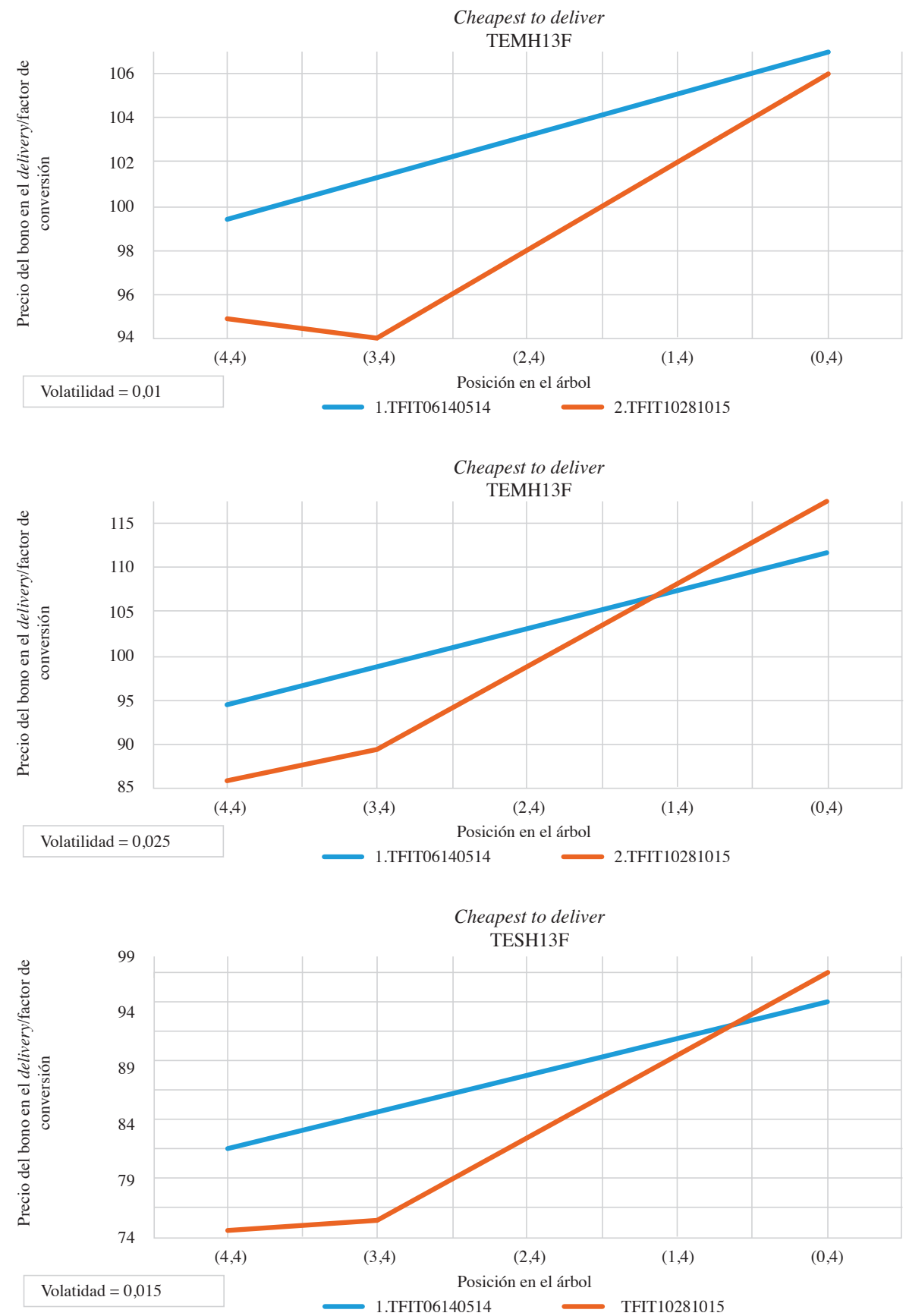

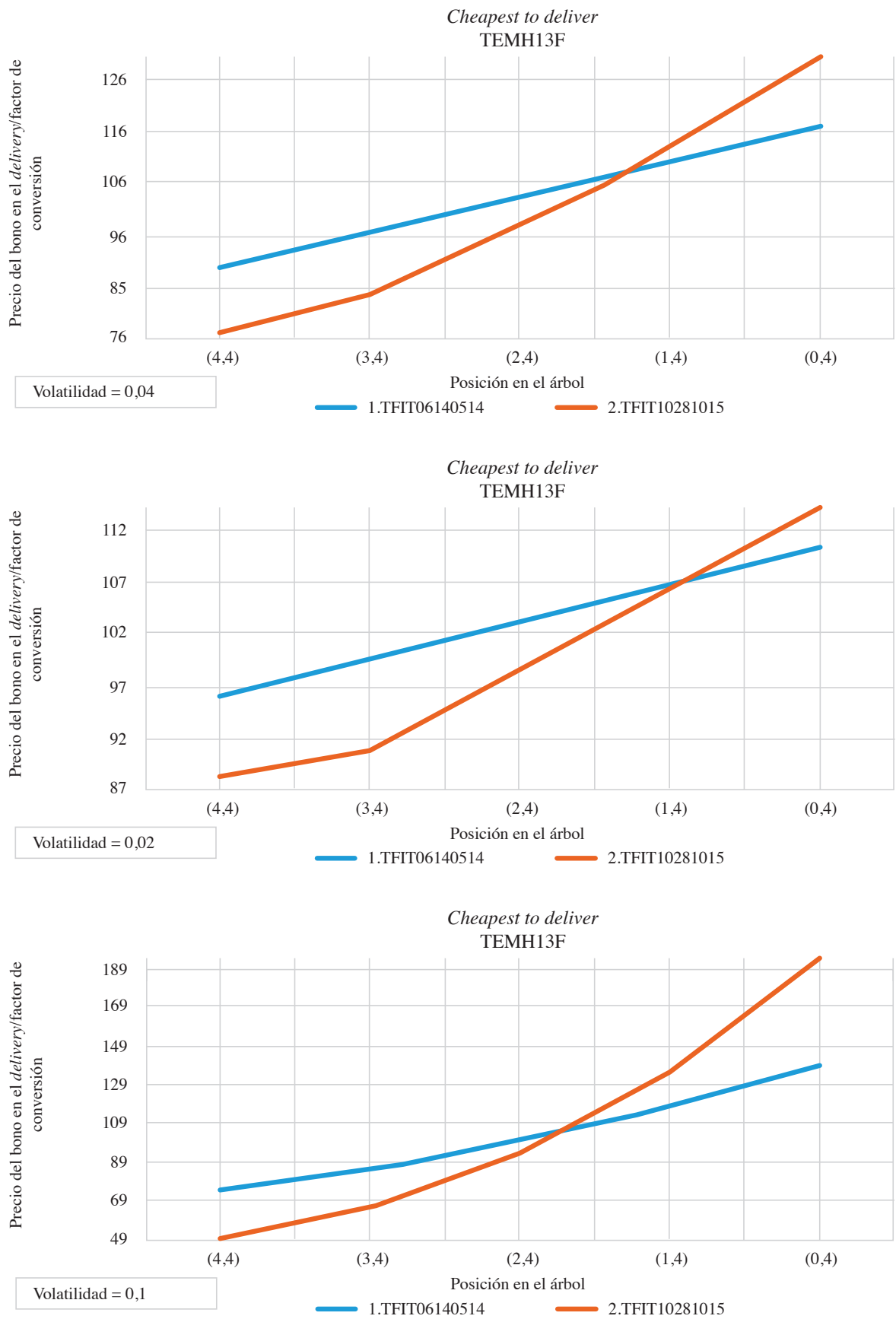


\section{Anexo 3}

Paso a paso de la implementación para el contrato de largo plazo: TELJ10F

Antes de implementar la metodología, y con el objeto de identificar si el CTD cambia para diferentes tasas, se calculó el precio del futuro en la fecha actual para distintos niveles de tasa (yield). En la figura 11 se observa que el entregable TFIT15240720 es el CTD cuando la tasa disminuye, mientras que el TFIT16240724 se convierte en el CTD cuando la tasa aumenta.

Figura 11: Precio del futuro vs. movimientos en las tasas para el contrato TELJ10F

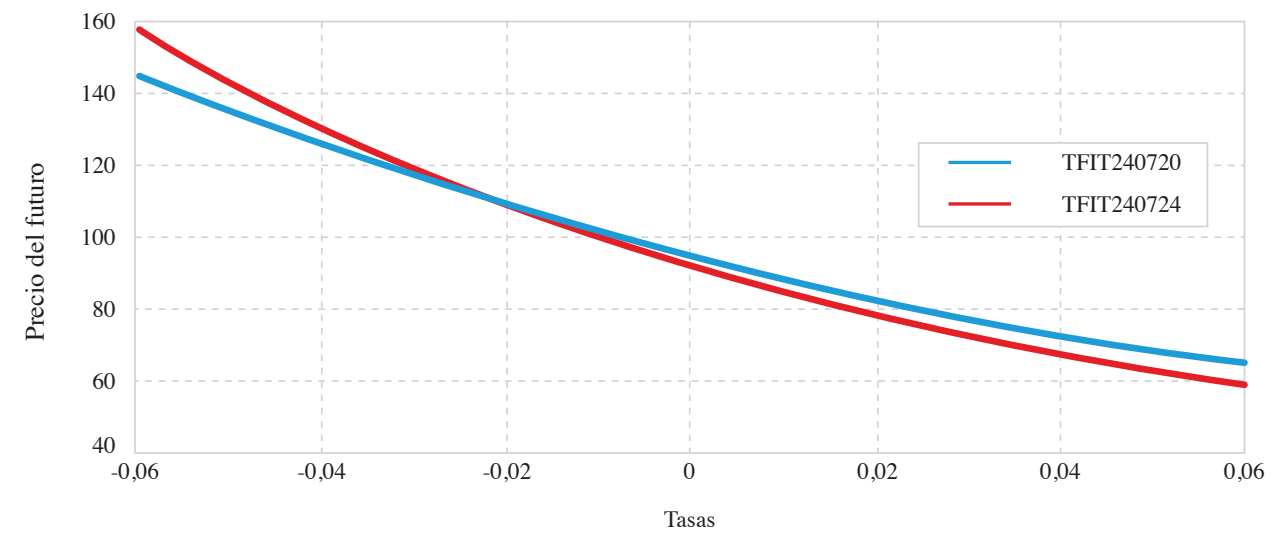

Tabla 27: Información para construir la curva cero cupón

\begin{tabular}{|c|c|}
\hline \multicolumn{2}{|c|}{ Factores de conversión de entregables } \\
\hline IBR overnight & 0,03455 \\
\hline IBR 30 días & 0,03345 \\
\hline
\end{tabular}

\begin{tabular}{|c|c|c|c|c|}
\hline Títulos del mercado & Precios de cierrre & Cupón & Fecha de vencimiento & Días al vencimiento \\
\hline TFIT05241110 & 102,891 & 7,5 & $24 / 11 / 2010$ & 286 \\
\hline TFIT04150812 & 106,952 & 9,25 & $15 / 08 / 2012$ & 916 \\
\hline TFIT04170413 & 96,95 & 6 & $17 / 04 / 2013$ & 1161 \\
\hline TFIT06141113 & 109,353 & 10,25 & $14 / 11 / 2013$ & 1372 \\
\hline TFIT06140514 & 105,925 & 9,25 & $14 / 05 / 2014$ & 1553 \\
\hline TFIT04180511 & 107,546 & 11 & $18 / 05 / 2016$ & 2288 \\
\hline TFIT15240720 & 114,754 & 11 & $24 / 07 / 2020$ & 3816 \\
\hline TFIT16240724 & 107,955 & 10 & $24 / 07 / 2024$ & 5277 \\
\hline
\end{tabular}

Fuente: elaboración propia. 
La curva cero cupón calculada para este caso tiene la siguiente forma (tabla 28):

Tabla 28: Curva cero cupón - Contrato TELJ10F

\begin{tabular}{|c|c|c|}
\hline Años al vencimiento & Días al vencimiento & Tasa \\
\hline 0,003 & 1 & 0,0348310 \\
\hline 0,082 & 30 & 0,0331793 \\
\hline 0,784 & 286 & 0,0360300 \\
\hline 2,510 & 916 & 0,0607068 \\
\hline 3,181 & 1161 & 0,0698531 \\
\hline 3,759 & 1372 & 0,0727735 \\
\hline 4,225 & 1553 & 0,0749812 \\
\hline 6,268 & 2288 & 0,0957451 \\
\hline 10,455 & 3816 & 0,0851759 \\
\hline 14,458 & 5277 & 0,0882242 \\
\hline
\end{tabular}

Fuente: elaboración propia.

Figura 12: Curva cero cupón - Contrato TELJ10F

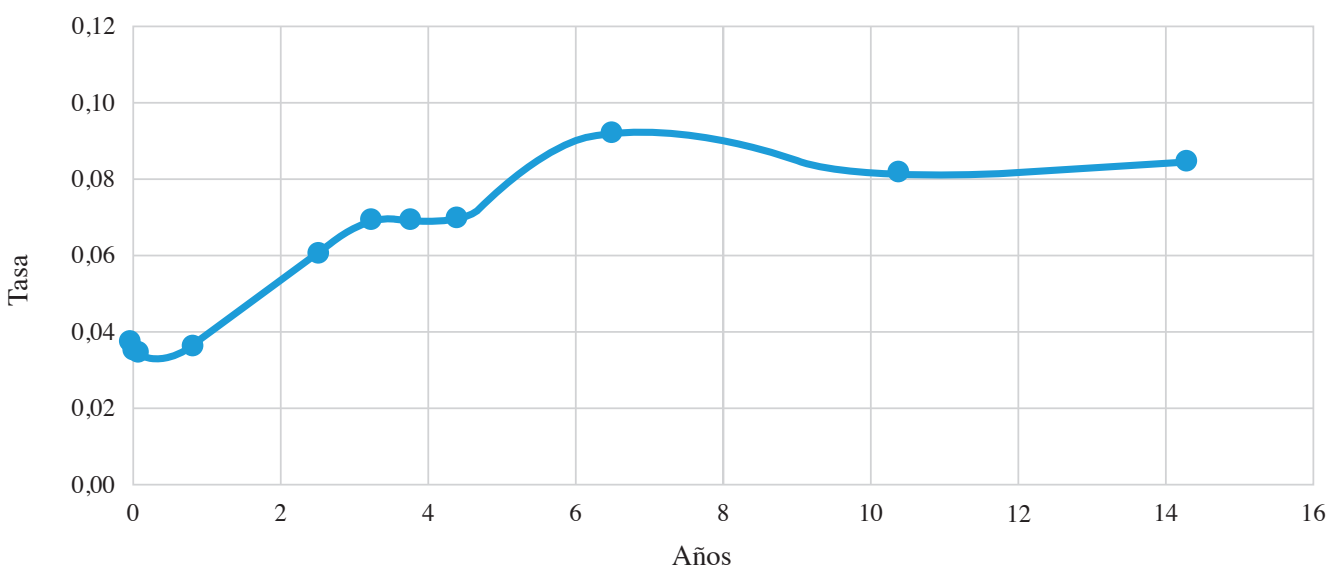

En la construcción de la curva cero cupón se incluyó información del mercado monetario, específicamente las tasas del mercado ors para los plazos de 1 y 30 . Esto con el fin de tener referencias de tasas de corto plazo que permitan realizar interpolación para hallar las tasas de los dos primeros pasos del árbol Ho-Lee. 
Tabla 29: Información para la construcción del árbol Ho-Lee

\begin{tabular}{|c|c|}
\hline Número de días desde la fecha actual hasta la fecha del delivery & 53 \\
\hline Número de pasos del árbol & 4 \\
\hline Número de días de cada periodo & 13,25 \\
\hline Volatilidad & 0,004254 \\
\hline Delta & 0,999717131 \\
\hline
\end{tabular}

Fuente: elaboración propia.

Con base en la CCC calculada, y utilizando el método de interpolación log linear on the discount factors (ecuación 3), se calculan las tasas cero cupón para los respectivos nodos del árbol (tabla 30):

Tabla 30: Cálculos intermedios en la construcción del árbol de tasas de Ho-Lee

\begin{tabular}{|c|c|c|}
\hline Pasos & Días & Tasas cero cupón \\
\hline 1 & 13,25 & 0,03325 \\
\hline 2 & 26,50 & 0,03319 \\
\hline 3 & 39,75 & 0,03396 \\
\hline 4 & 53,00 & 0,03456 \\
\hline 5 & 66,25 & 0,03492 \\
\hline
\end{tabular}

\begin{tabular}{|c|c|}
\hline Pasos & Precio del bono P(n) \\
\hline 1 & 0,99879 \\
\hline 2 & 0,99759 \\
\hline 3 & 0,99631 \\
\hline 4 & 0,99499 \\
\hline 5 & 0,99368 \\
\hline
\end{tabular}

Tabla 31: Árbol de tasas por medio de metodología Ho-Lee

\begin{tabular}{|c|c|c|c|c|c|c|c|c|}
\hline Hoy & & 1 & & 2 & & 3 & & 4 \\
\hline \multirow[t]{5}{*}{$3,325 \%$} & 1 & $3,702 \%$ & 2 & $4,330 \%$ & 3 & $4,806 \%$ & 4 & $5,196 \%$ \\
\hline & 0 & $2,923 \%$ & 1 & $3,551 \%$ & 2 & $4,026 \%$ & 3 & $4,416 \%$ \\
\hline & & & 0 & $2,772 \%$ & 1 & $3,247 \%$ & 2 & $3,637 \%$ \\
\hline & & & & & 0 & $2,468 \%$ & 1 & $2,858 \%$ \\
\hline & & & & & & & 0 & $2,078 \%$ \\
\hline
\end{tabular}

Fuente: elaboración propia. 
A continuación se presentan los resultados de los factores de descuento, las tasas equivalentes y los precios del futuro utilizando la misma volatilidad calculada como la mayor desviación estándar de las tasas de retorno históricas de los dos entregables.

Tabla 32: Factores de descuento para cada flujo y posición en el árbol - Contrato TELJ10F

\begin{tabular}{|c|c|c|c|c|c|c|}
\hline \multicolumn{7}{|c|}{ Factores de descuento } \\
\hline & & \multicolumn{5}{|c|}{ Posición en el árbol } \\
\hline & Flujos & $(4,4)$ & $(3,4)$ & $(2,4)$ & $(1,4)$ & $(0,4)$ \\
\hline \multirow[t]{12}{*}{ TFIT15240720 } & 1 & 0,98395 & 0,98626 & 0,98858 & 0,99090 & 0,99323 \\
\hline & 2 & 0,91072 & 0,92001 & 0,92939 & 0,93886 & 0,94843 \\
\hline & 3 & 0,83271 & 0,84779 & 0,86316 & 0,87880 & 0,89472 \\
\hline & 4 & 0,74192 & 0,76128 & 0,78113 & 0,80151 & 0,82242 \\
\hline & 5 & 0,65964 & 0,68214 & 0,70541 & 0,72947 & 0,75436 \\
\hline & 6 & 0,56378 & 0,58757 & 0,61237 & 0,63821 & 0,66515 \\
\hline & 7 & 0,48790 & 0,51247 & 0,53829 & 0,56541 & 0,59390 \\
\hline & 8 & 0,44735 & 0,47356 & 0,50131 & 0,53068 & 0,56178 \\
\hline & 9 & 0,41017 & 0,43760 & 0,46687 & 0,49809 & 0,53140 \\
\hline & 10 & 0,37608 & 0,40437 & 0,43479 & 0,46749 & 0,50266 \\
\hline & 11 & 0,34474 & 0,37358 & 0,40483 & 0,43870 & 0,47540 \\
\hline & Flujos & $(4,4)$ & $(3,4)$ & $(2,4)$ & $(1,4)$ & $(0,4)$ \\
\hline \multirow[t]{11}{*}{ TFIT16240724 } & 1 & 0,9839 & 0,9863 & 0,9886 & 0,9909 & 0,9932 \\
\hline & 2 & 0,9107 & 0,9200 & 0,9294 & 0,9389 & 0,9484 \\
\hline & 3 & 0,8327 & 0,8478 & 0,8632 & 0,8788 & 0,8947 \\
\hline & 4 & 0,7419 & 0,7613 & 0,7811 & 0,8015 & 0,8224 \\
\hline & 5 & 0,6596 & 0,6821 & 0,7054 & 0,7295 & 0,7544 \\
\hline & 6 & 0,5638 & 0,5876 & 0,6124 & 0,6382 & 0,6651 \\
\hline & 7 & 0,4879 & 0,5125 & 0,5383 & 0,5654 & 0,5939 \\
\hline & 8 & 0,4473 & 0,4736 & 0,5013 & 0,5307 & 0,5618 \\
\hline & 9 & 0,4102 & 0,4376 & 0,4669 & 0,4981 & 0,5314 \\
\hline & 10 & 0,3761 & 0,4044 & 0,4348 & 0,4675 & 0,5027 \\
\hline & 11 & 0,3447 & 0,3736 & 0,4048 & 0,4387 & 0,4754 \\
\hline
\end{tabular}




\begin{tabular}{|c|c|c|c|c|c|c|}
\hline & 12 & 0,3077 & 0,3361 & 0,3670 & 0,4008 & 0,4378 \\
\cline { 2 - 7 } & 13 & 0,2747 & 0,3023 & 0,3328 & 0,3663 & 0,4031 \\
\cline { 2 - 7 } & 14 & 0,2452 & 0,2720 & 0,3017 & 0,3347 & 0,3713 \\
\cline { 2 - 7 } & 15 & 0,2207 & 0,2467 & 0,2758 & 0,3083 & 0,3447 \\
\hline
\end{tabular}

Fuente: elaboración propia.

A partir de los factores de descuentos de la tabla 32 es posible calcular las tasas equivalentes y con ellas construir una curva de tasas para cada nodo final del árbol. Estas curvas se extienden desde la fecha del delivery hasta la fecha del último flujo de los entregables del contrato de futuro. Dichas tasas se calcularon usando la ecuación 29.

Tabla 33: Árbol de tasas Ho-Lee - Contrato TELJ10F
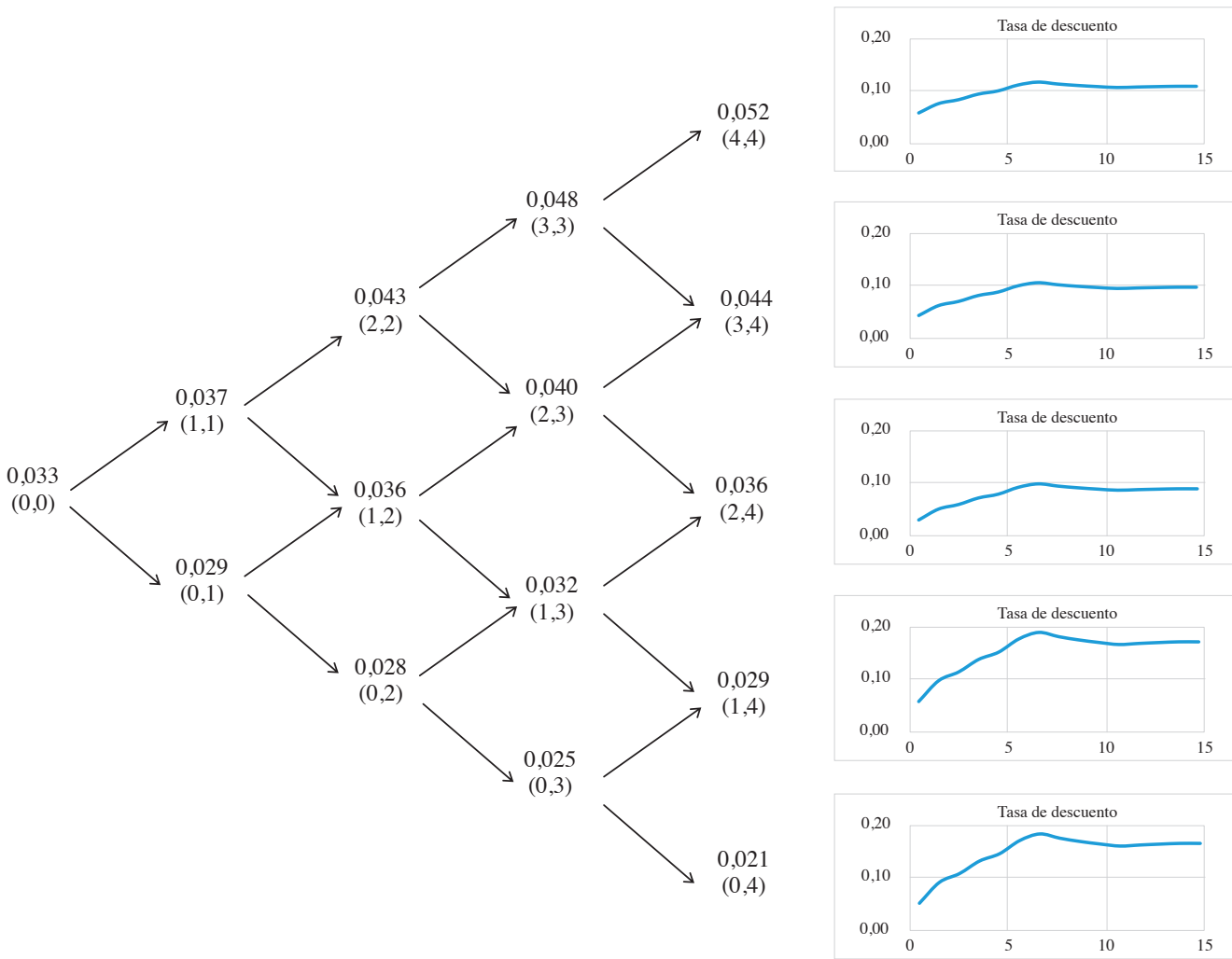

Fuente: elaboración propia. 
Tabla 34: Precio de los bonos entregables en la fecha del delivery - Contrato TELJ10F

\begin{tabular}{|c|c|c|c|c|c|c|}
\hline \multicolumn{7}{|c|}{ Precio de los bonos en la fecha del delivery } \\
\hline Fecha actual & \multicolumn{2}{|r|}{$11 / 02 / 10$} & & & & \\
\hline Última fecha que pagó cupón & \multicolumn{2}{|r|}{$24 / 07 / 09$} & & & & \\
\hline Cupón & \multicolumn{2}{|r|}{11} & & & & \\
\hline Número de días & \multicolumn{2}{|r|}{202} & & & & \\
\hline \multirow[t]{3}{*}{ Intereses devengados } & \multicolumn{2}{|r|}{6,08767123} & & & & \\
\hline & & \multicolumn{5}{|c|}{ Posición en el árbol } \\
\hline & Flujos & $(4,4)$ & $(3,4)$ & $(2,4)$ & $(1,4)$ & $(0,4)$ \\
\hline \multirow[t]{11}{*}{ TFIT15240720 } & 1 & 10,82341 & 10,84886 & 10,87437 & 10,89994 & 10,92557 \\
\hline & 2 & 10,01796 & 10,12008 & 10,22324 & 10,32745 & 10,43272 \\
\hline & 3 & 9,15977 & 9,32574 & 9,49471 & 9,66675 & 9,84190 \\
\hline & 4 & 8,16116 & 8,37404 & 8,59247 & 8,81660 & 9,04658 \\
\hline & 5 & 7,25603 & 7,50355 & 7,75951 & 8,02421 & 8,29793 \\
\hline & 6 & 6,20158 & 6,46330 & 6,73607 & 7,02036 & 7,31664 \\
\hline & 7 & 5,36685 & 5,63722 & 5,92122 & 6,21953 & 6,53287 \\
\hline & 8 & 4,92081 & 5,20916 & 5,51440 & 5,83753 & 6,17960 \\
\hline & 9 & 4,51184 & 4,81359 & 5,13552 & 5,47898 & 5,84541 \\
\hline & 10 & 4,13684 & 4,44804 & 4,78265 & 5,14244 & 5,52928 \\
\hline & 11 & 38,26576 & 41,46716 & 44,93639 & 48,69586 & 52,76985 \\
\hline Precio Sucio & & 108,82201 & 114,21074 & 119,97056 & 126,12963 & 132,71834 \\
\hline Precio Limpio & & 102,73434 & 108,12307 & 113,88289 & 120,04196 & 126,63067 \\
\hline Fecha actual & \multicolumn{2}{|c|}{$11 / 02 / 10$} & & & & \\
\hline Última fecha que pagó cupón & \multicolumn{2}{|c|}{$24 / 07 / 09$} & & & & \\
\hline Cupón & \multicolumn{2}{|c|}{10} & & & & \\
\hline Número de días & \multicolumn{2}{|c|}{202} & & & & \\
\hline \multirow[t]{2}{*}{ Intereses devengados } & \multicolumn{2}{|c|}{5,53424658} & & & & \\
\hline & Flujos & $(4,4)$ & $(3,4)$ & $(2,4)$ & $(1,4)$ & $(0,4)$ \\
\hline \multirow[t]{3}{*}{ TFIT16240724 } & 1 & 9,8395 & 9,8626 & 9,8858 & 9,9090 & 9,9323 \\
\hline & 2 & 9,1072 & 9,2001 & 9,2939 & 9,3886 & 9,4843 \\
\hline & 3 & 8,3271 & 8,4779 & 8,6316 & 8,7880 & 8,9472 \\
\hline
\end{tabular}




\begin{tabular}{|c|c|c|c|c|c|c|}
\hline & 4 & 7,4192 & 7,6128 & 7,8113 & 8,0151 & 8,2242 \\
\cline { 2 - 7 } & 5 & 6,5964 & 6,8214 & 7,0541 & 7,2947 & 7,5436 \\
\cline { 2 - 7 } & 6 & 5,6378 & 5,8757 & 6,1237 & 6,3821 & 6,6515 \\
\cline { 2 - 7 } & 7 & 4,8790 & 5,1247 & 5,3829 & 5,6541 & 5,9390 \\
\cline { 2 - 7 } & 8 & 4,4735 & 4,7356 & 5,0131 & 5,3068 & 5,6178 \\
\cline { 2 - 7 } & 9 & 4,1017 & 4,3760 & 4,6687 & 4,9809 & 5,3140 \\
\cline { 2 - 7 } & 10 & 3,7608 & 4,0437 & 4,3479 & 4,6749 & 5,0266 \\
\cline { 2 - 7 } & 11 & 3,4474 & 3,7358 & 4,0483 & 4,3870 & 4,7540 \\
\cline { 2 - 7 } & 12 & 3,0771 & 3,3607 & 3,6703 & 4,0085 & 4,3778 \\
\cline { 2 - 7 } & 13 & 2,7466 & 3,0232 & 3,3276 & 3,6626 & 4,0314 \\
\cline { 2 - 7 } & 14 & 2,4521 & 2,7202 & 3,0175 & 3,3473 & 3,7131 \\
\hline Precio Sucio & & 24,2715 & 27,1355 & 30,3375 & 33,9173 & 37,9195 \\
\hline Precio Limpio & & 94,60260 & 100,57160 & 107,07983 & 114,18280 & 121,94211 \\
\hline
\end{tabular}

Fuente: elaboración propia.

Tabla 35: Precio del CTD en la fecha del delivery - Contrato TELJ10F

\begin{tabular}{|c|c|c|c|c|c|c|c|c|}
\hline \multicolumn{7}{|c|}{ Precio de los bonos en la fecha del delivery } \\
\hline \multicolumn{2}{|c|}{} & \multicolumn{7}{c|}{ Posición en el árbol } \\
\cline { 4 - 9 } & $(4,4)$ & $(3,4)$ & $(2,4)$ & $(1,4)$ & $(0,4)$ \\
\hline 1. TFIT15240720 & Precios & & 102,734 & 108,123 & 113,883 & 120,042 & 126,631 \\
\hline & FC & 1,204057 & \multicolumn{7}{|c|}{} \\
\cline { 2 - 9 } & Precio del Futuro & & 85,323 & 89,799 & 94,583 & 99,698 & 105,170 \\
\hline 2. TFIT16240724 & Precios & & 94,603 & 100,572 & 107,080 & 114,183 & 121,942 \\
\hline & FC & 1,165523 & & & & & \\
\cline { 2 - 9 } & Precio del Futuro & & 81,168 & 86,289 & 91,873 & 97,967 & 104,624 \\
\hline & CTD BONO CTD & & 81,168 & 86,289 & 91,873 & 97,967 & 104,624 \\
\hline
\end{tabular}

Fuente: elaboración propia. 
Tabla 36: Árbol de precios del futuro - Contrato TELJ10F

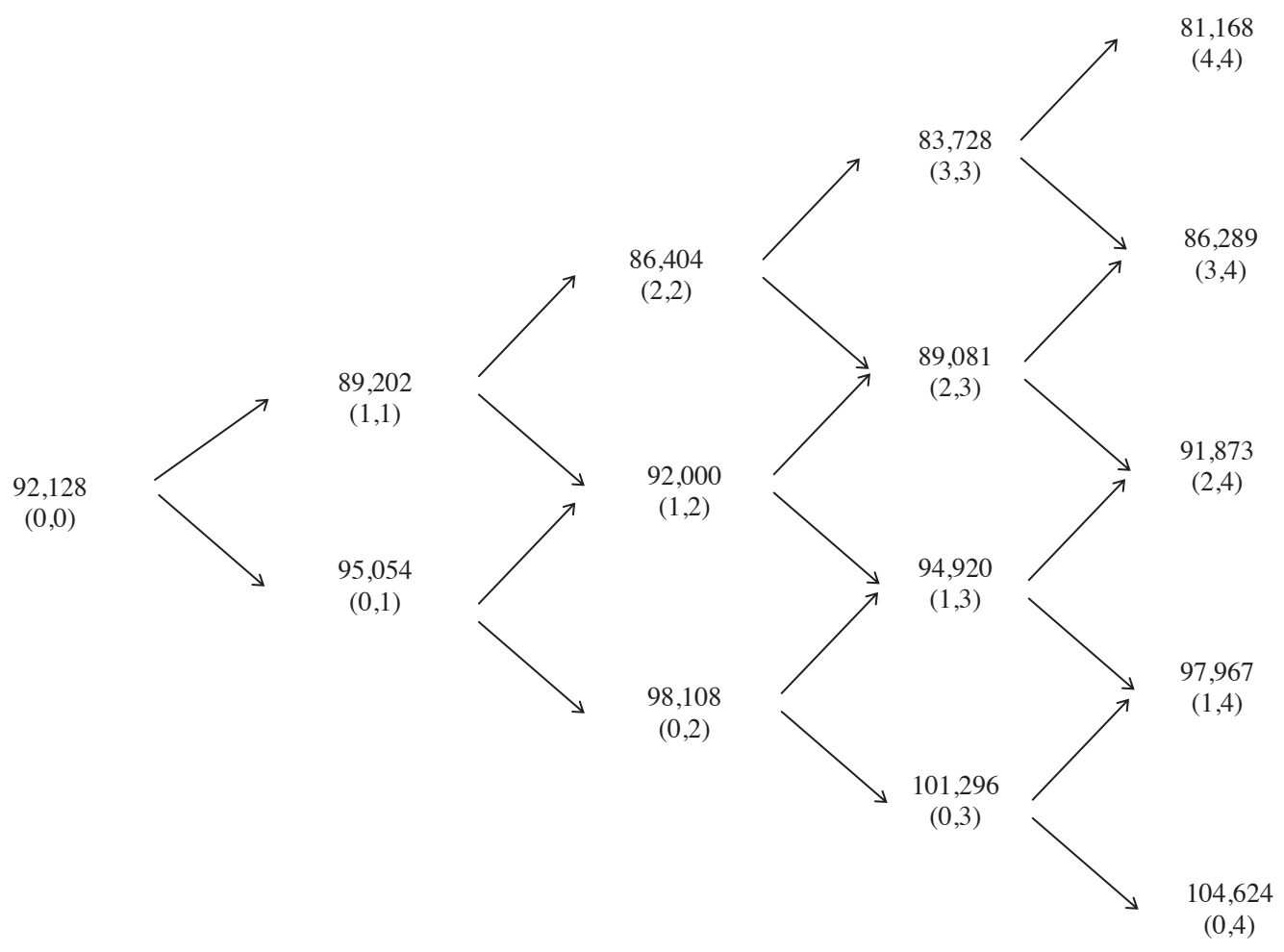

Fuente: elaboración propia.

Dado que el CTD en todos los nodos del árbol el día del delivery es el mismo título, no se precisa calcular el árbol de probabilidades de que un determinado entregable se convierta en el CTD en la fecha actual. 
Figura 13: Análisis de sensibilidad del CTD ante cambios en la curva cero cupón - Contrato TELJ10F
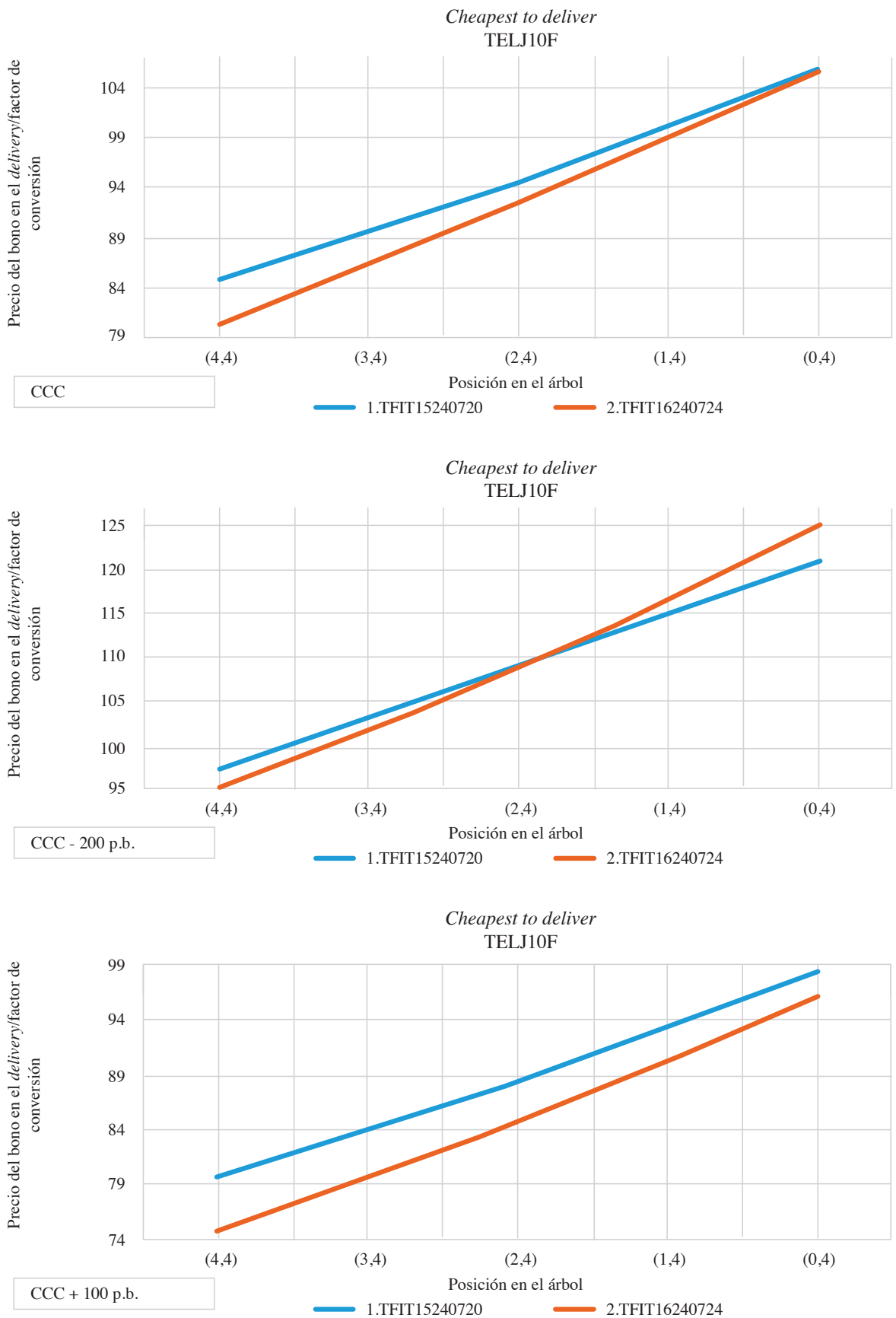

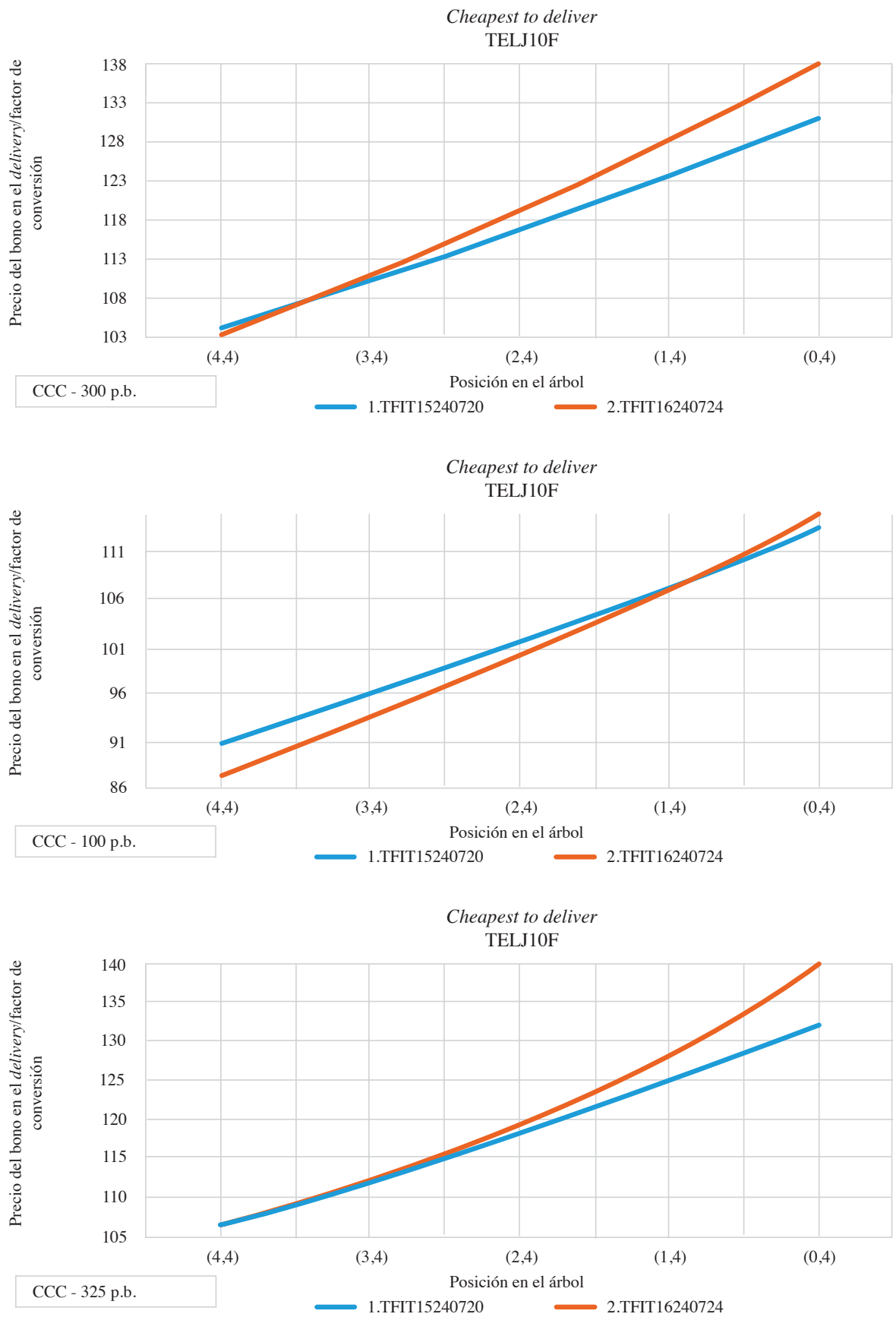
Figura 14: Análisis de sensibilidad del CTD ante cambios en la volatilidad - Contrato TELJ10F
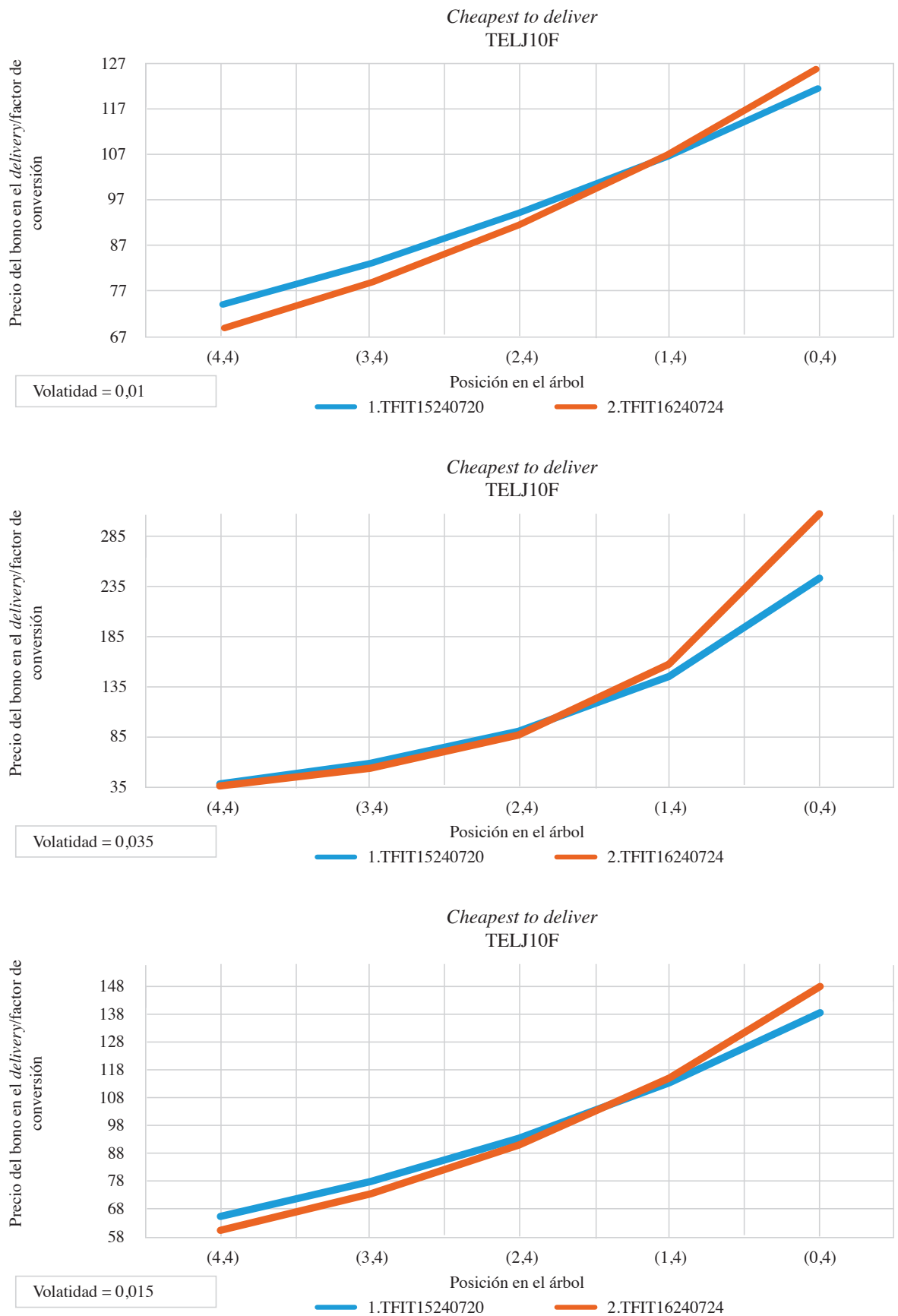

ODEON, ISSN: 1794-1113, E-ISSN: 2346-2140, N 15, julio-diciembre de 2018, pp. 73-137 

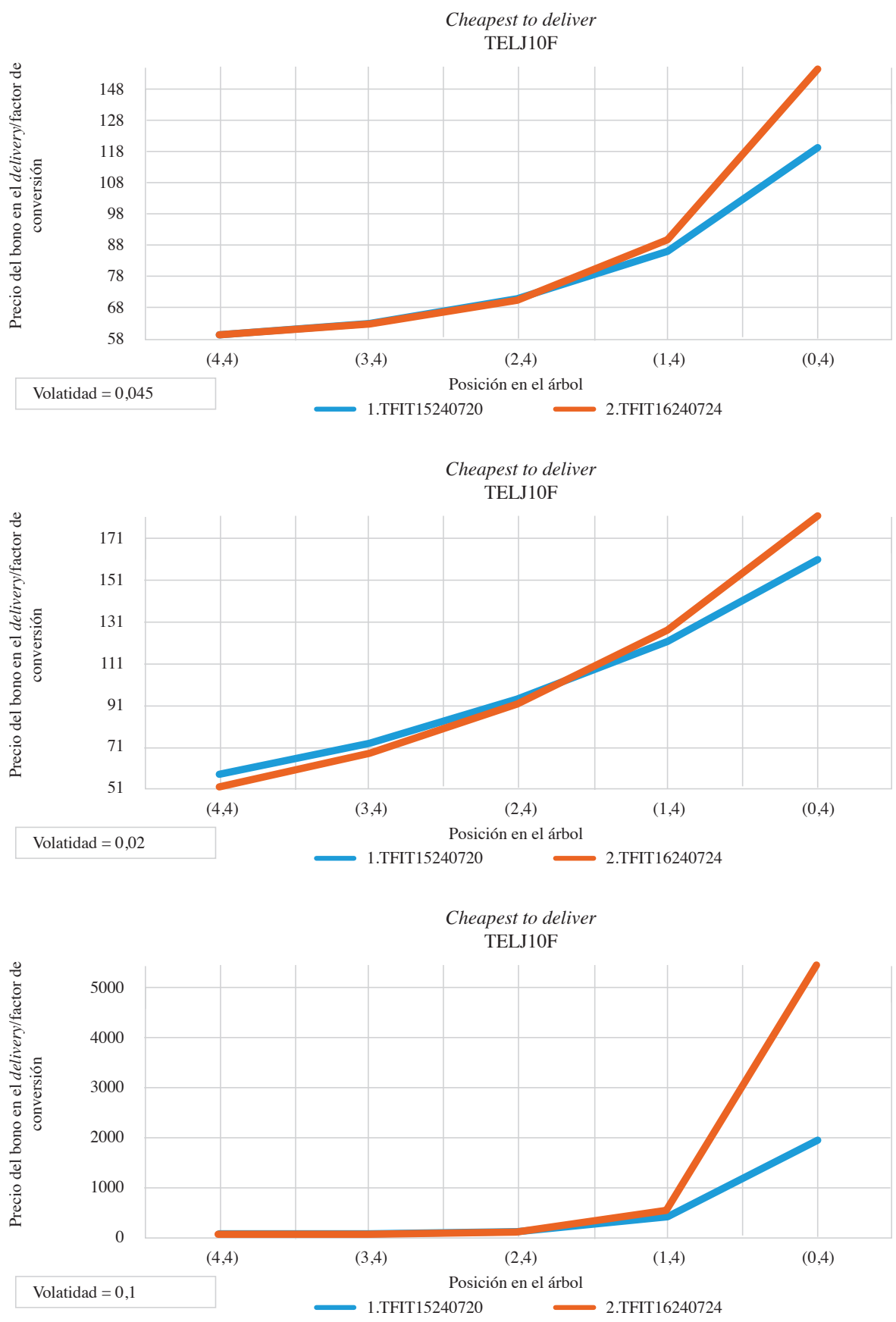